\title{
UK Renal Registry 19th Annual Report: Chapter 12 Multisite Dialysis Access Audit in England, Northern Ireland and Wales in 2015 and 2014 PD One Year Follow-up: National and Centre-specific Analyses
}

\author{
Barnaby Hole ${ }^{\mathrm{abc}}$, Fergus Caskey ${ }^{\mathrm{abc}}$, Katharine Evans ${ }^{\mathrm{a}}$, Richard Fluck ${ }^{\mathrm{d}}$, \\ Mick Kumwenda ${ }^{e}$, Retha Steenkamp ${ }^{a}$, Martin Wilkie ${ }^{f}$ \\ aUK Renal Registry, Bristol, UK; ${ }^{b}$ University of Bristol, Bristol, UK; ${ }^{c}$ North Bristol NHS Trust, Bristol, UK;

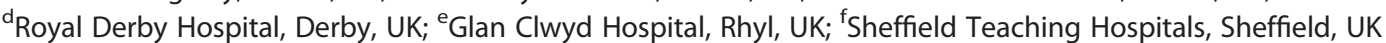

\section{Keywords}

Chronic kidney disease - Diabetes - Dialysis - End stage renal disease $\cdot$ Established renal failure $\cdot$ Haemodialysis - Peritoneal dialysis - Prevalence - Renal replacement therapy - Transplantation $\cdot$ Treatment modality $\cdot$ Vascular access

\section{Summary}

- Data are presented from the fifth combined vascular and peritoneal dialysis (PD) access audit.

- In 2015, 53 of 62 centres in England, Wales and Northern Ireland returned data on first access for 4,032 incident haemodialysis (HD) and 1,075 incident PD recipients.

- Of the 5,107 incident patients, $21.0 \%$ started PD, $30.3 \%$ started HD with an arteriovenous fistula (AVF) or graft (AVG), 28.7\% with a tunnelled line (TL) and $19.9 \%$ with a non-tunnelled line (NTL).

- Wide variation in definitive access use (defined as primary AVF, AVG or PD) was apparent between centres.

\section{KARGER}

Fax +4161306 1234 E-Mail karger@karger.com www.karger.com/nef
C 2017 The UK Renal Registry

Published by S. Karger AG, Base

Karger
Open access

This article is licensed under the Creative Commons AttributionNonCommercial-NoDerivatives 4.0 International License (CC BYNC-ND) (http://www.karger.com/Services/OpenAccessLicense). Usage and distribution for commercial purposes as well as any distribution of modified material requires written permission.
- Only 10 centres achieved the $60 \%$ target for AVF/ AVG use amongst incident HD recipients.

- Seventeen centres achieved the 80\% target for AVF/ AVG/PD use amongst prevalent dialysis recipients.

- Timely presentation to a nephrologist and referral to a dialysis access surgeon were key determinants of the likelihood of definitive access:

- $60.0 \%$ of patients known to a nephrologist for over 90 days initiated dialysis with definitive access compared with $15.2 \%$ of those who were known to a nephrologist for 90 days or less.

- Among incident HD patients who were reviewed by a surgeon three months prior to starting dialysis, AVF/AVG use was 70.9\% compared with $10.0 \%$ in those who were not.

- AVF/AVG use amongst incident HD recipients increased with rising age and body mass index (BMI). This was due to lower rates of PD and preemptive transplant (PTx) amongst older patients and the obese.

- In centres that placed non-surgical PD catheters, $25.9 \%$ of incident renal replacement therapy (RRT) patients started PD, compared with $21.0 \%$ overall. 
- For centres returning data on one-year PD access outcomes, $76.6 \%$ of patients starting PD continued to use this modality one year later after censoring for death, withdrawal from dialysis and transplantation.

- The median one-year PD catheter failure rate was $13.3 \%$.

- This report demonstrates wide variation in practice between centres across several domains in the provision of dialysis access. Further work is required to understand the underlying reasons.

\section{Introduction}

Provision of definitive dialysis access is an important measure of good clinical care for patients with established renal failure. Relevant recommendations and audit standards are presented in the Renal Association clinical practice guidelines (table 12.1). The annual multisite dialysis access audit provides centre-level information on access provision in England, Wales and Northern Ireland. Although the Renal Association undertook a national vascular access audit in 2005, published with outcomes data in 2012 by the UK Renal Registry (UKRR) [1], this is the fifth annual audit that combines peritoneal and vascular access, presenting information for patients starting dialysis between 1st January and 31st December 2015.

One objective of this audit has been to highlight centre-level performance variation and explore factors that may contribute to the provision of high quality vascular and peritoneal access. For the 19th Annual Report, this chapter is expanded to allow more detailed examination of dialysis access patterns through the incorporation of UKRR data. The resulting improved data completeness allowed more detailed analysis and data presentation, for example, permitting analysis of the relationship between dialysis access and PTx. The chapter is presented in two parts: part 1 presents detailed data from the fifth multisite dialysis access

Table 12.1. Summary of relevant audit standards stated in the Renal Association (RA) clinical practice guidelines

\begin{tabular}{|c|c|c|c|}
\hline \multicolumn{2}{|c|}{ RA audit measure/guideline* } & \multirow{2}{*}{$\frac{\text { Reported }}{\text { Yes }}$} & \multirow{2}{*}{$\begin{array}{c}\text { Notes } \\
\text { Table } 12.3 \\
\text { Table } 12.4 \\
\text { Table } 12.9 \\
\text { Table } 12.10 \\
\end{array}$} \\
\hline 1 & $\begin{array}{l}\text { Proportion of planned renal replacement therapy initiations with established } \\
\text { access or pre-emptive transplantation (no minimum audit standard) }\end{array}$ & & \\
\hline 2 & $\begin{array}{l}60 \% \text { of all incident patients with established end stage kidney disease } \\
\text { commencing planned haemodialysis should receive dialysis via a functioning } \\
\text { arteriovenous fistula or arteriovenous graft }\end{array}$ & Yes & $\begin{array}{c}\text { Table } 12.3 \\
\text { Table } 12.4 \\
\text { Table } 12.9 \\
\text { Table } 12.10 \\
\text { Figure } 12.9\end{array}$ \\
\hline 3 & $\begin{array}{l}80 \% \text { of all prevalent long-term dialysis patients should receive dialysis } \\
\text { treatment via 'definitive access': arteriovenous fistula, arteriovenous graft or } \\
\text { peritoneal dialysis }\end{array}$ & Yes & $\begin{array}{l}\text { Figure } 12.11 \\
\text { Table } 12.10\end{array}$ \\
\hline 4 & $\begin{array}{l}\text { Catheter patency - more than } 80 \% \text { of catheters should be patent at one year } \\
\text { (censoring for death and elective modality change) }\end{array}$ & Yes & $\begin{array}{l}\text { Figure } 12.17 \\
\text { Figure } 12.19\end{array}$ \\
\hline 5 & Complications following peritoneal dialysis catheter insertion: & Partly & $\begin{array}{l}\text { Figure } 12.18 \\
\text { Figure } 12.19\end{array}$ \\
\hline $5 \mathrm{a}$ & Bowel perforation $<1 \%$ & No & Not captured by the audit \\
\hline $5 b$ & Significant haemorrhage $<1 \%$ & No & Not captured by the audit \\
\hline $5 c$ & Exit site infection within two weeks of catheter insertion $<5 \%$ & No & Not captured by the audit \\
\hline $5 \mathrm{~d}$ & Peritonitis within two weeks of catheter insertion $<5 \%$ & No & Low data completeness \\
\hline $5 \mathrm{e}$ & $\begin{array}{l}\text { Functional catheter problem requiring manipulation or replacement or leading } \\
\text { to technique failure }<20 \%\end{array}$ & No & Not captured by the audit \\
\hline
\end{tabular}

\footnotetext{
*Audit standards from the most recent Renal Association guidelines (June 2017) are presented. Current and previous guidelines are available on the Renal Association website (http://www.renal.org/guidelines/current-guidelines)
} 
audit; and part 2 presents summary data over the five years since the annual collection was started in 2011.

The term 'established renal failure' used within this chapter is synonymous with the terms 'end stage renal failure' and 'end stage kidney disease'. These alternative terms are in widespread international use, but are less acceptable to patients.

\section{Methods}

In 2016, all adult renal centres in England, Wales and Northern Ireland were asked to provide vascular and peritoneal access data for incident (1st January to 31st December 2015) and prevalent dialysis patients. Access data for incident patients were collected at patient level, whereas centre-level data were submitted for prevalent patients. Table 12.2 presents a full glossary of collected variables. Data were collected using Microsoft Excel spreadsheets circulated by the UKRR.

Table 12.2. Glossary of variables collected in the 2015 multisite dialysis access audit

\begin{tabular}{|c|c|c|}
\hline Audit data item & Definition [format] & $\mathrm{PD} / \mathrm{HD}$ or both \\
\hline NHS number & NHS number (England \& Wales) [numerical] & Both \\
\hline Forename & {$[$ text $]$} & Both \\
\hline DoB & Date of birth [DD/MM/YY] & Both \\
\hline Gender & [Male/Female/Unknown] & Both \\
\hline Postcode & The postcode of the patient's usual address [alpha-numerical] & Both \\
\hline First RRT treatment centre code & $\begin{array}{l}\text { Renal treatment centre where first dialysis took place } \\
\text { [treatment centre ID code] }\end{array}$ & Both \\
\hline Primary renal diagnosis & Primary renal diagnosis [EDTA four digit diagnosis code] & Both \\
\hline BMI & $\begin{array}{l}\text { BMI at time of access insertion (weight in } \mathrm{kg} / \text { height in } \mathrm{m}^{2} \text { ) } \\
\text { [numerical] }\end{array}$ & Both \\
\hline Was an AVF/AVG attempted before 1st dialysis? & $\begin{array}{l}\text { Was an AVF/AVG attempted before the first ever dialysis } \\
\text { session? [Yes/No/Unknown] }\end{array}$ & Both \\
\hline Date FIRST EVER dialysis session & Date of first ever dialysis session [DD/MM/YY] & Both \\
\hline First ever modality & First ever renal replacement modality [HD/PD] & Both \\
\hline Access in use at first ever dialysis & $\begin{array}{l}\text { Dialysis access in use at first dialysis (may not be first access } \\
\text { created) }[\mathrm{AVF} / \mathrm{AVG} / \text { vein loop/TL/NTL/PD/temporary } \\
\mathrm{PD} \text { catheter }]\end{array}$ & Both \\
\hline Access in use at three months & $\begin{array}{l}\text { Dialysis access in use three months after the start of first } \\
\text { treatment }[\mathrm{AVF} / \mathrm{AVG} / \mathrm{vein} \text { loop/TL/NTL/PD/temporary } \\
\mathrm{PD} \text { catheter/recovered/transplant/conservative/death/lost to } \\
\text { follow-up/transferred out] }\end{array}$ & Both \\
\hline Peritonitis episode & Peritonitis episode within two weeks of insertion? [Yes/No] & PD only \\
\hline Access complication & $\begin{array}{l}\text { Reason for access failure/discontinuation [selection from } \\
27 \text { item list] }\end{array}$ & Both \\
\hline Date of access failure/discontinuation & Date access is no longer usable for treatment [DD/MM/YY] & Both \\
\hline Comments & Any relevant comments [text] & Both \\
\hline
\end{tabular}

RRT - renal replacement therapy; BMI - body mass index; HD - haemodialysis; PD - peritoneal dialysis; AVF - arteriovenous fistula; AVG - arteriovenous graft; TL - tunnelled line; NTL - non-tunnelled line 
Records were validated against the UKRR database to confirm that the population collected at each centre for the audit was the same as, or representative of, the incident population at that centre collected via the routine quarterly return. Data checks were made by cross-referencing with the UKRR database. Any patients identified from the UKRR as not incident to dialysis between 1st January 2015 and 31st December 2015 were excluded. For the purposes of this audit, patients were categorised as having acute kidney injury (AKI) if their access at three months was recorded as 'recovered renal function' and were therefore excluded from analysis. Patients with missing information for access at start, age and date of starting RRT were excluded from the analysis. Patients were excluded when there was no matching record in the UKRR database (patient assumed to be AKI) and when aged $<18$ years. If a centre reported prevalent numbers that differed by more than $10 \%$ from those in the UKRR database, it was excluded. Cross-referencing also enabled ascertainment of mortality within three months of commencing dialysis.

Patients starting HD were grouped by type of first vascular access: arteriovenous fistula, arteriovenous graft, tunnelled dialysis line, non-tunnelled dialysis line. Patients starting PD were categorised by the insertion technique: open surgery, laparoscopic, peritoneoscopic or percutaneous. Access at three months was defined as the type of access in use at three months after starting dialysis. If a patient was no longer receiving dialysis at three months (but had not recovered renal function), the reason was recorded instead, for example, 'death' or 'transplantation'. Referral time was defined as the number of days between the date of first being seen by a renal physician (as an inpatient or outpatient) and the date of commencing dialysis. A patient was classified as presenting 'late' if they had a referral time of less than 90 days.

Access failure was defined when it was no longer usable for dialysis with the date and cause of access failure reported. For the purposes of analysis, HD access failure was grouped into five causes: maturation, mechanical, infection, other and unknown. PD technique failure was grouped into six causes: infection, catheter related, solute/water clearance, leaks/hernia, other and unknown. Access failure was censored for death, transplantation, withdrawal from RRT and elective switching of access type. It was the intention to only capture access failures relating to the first access that was performed. If the reason recorded for access failure was not related to the first type of access recorded, then the data were not included in this analysis.

Centres that reported data on PD patients in the 2014 vascular and peritoneal access audit were asked to complete a one year follow-up of their PD patients. Additional information was requested on the date of $\mathrm{PD}$ catheter failure, the reason for catheter failure, the number of catheters used during the year and the modality in use at one year after starting PD. Analyses that use these data are titled 'PD follow-up audit'.

For the first time this chapter includes data for PTx recipients. This reflects the amended (2015) Renal Association guidelines for planned RRT initiation, which include PTx in the audit standard (table 12.1). Where possible, these data have been included at centre level to aid in the interpretation of the effects of PTx upon rates of definitive and non-definitive dialysis access. Transplant and non-transplant centres work together to prepare patients for PTx, but for the purpose of these analyses, patients have been allocated to their most likely treatment centre (transplant or non-transplant) using the approach of Judge et al. [2]; this is based on patient postcode and the likelihood of receiving care in a centre.

Separate and combined analyses were performed for incident $\mathrm{HD}$ and PD patients as appropriate. Due to the exploratory nature of the audit the analyses have been limited to descriptive statistics of frequencies, percentages and unadjusted associations between variables. Centre-to-centre performance comparisons are made in the context of varying patient demography, case mix and volume. If a centre had $>50 \%$ missing returns for a particular data field, then all patients from that centre were excluded from analyses involving that data field. The data were analysed using SAS 9.3.

\section{Part 1 - Results from the 2015 Multisite Dialysis Access audit}

Of 62 centres contacted, 53 returned data on first dialysis access and data from 52 centres were used. After individual patient exclusions, 5,107 patients were included, comprising 4,032 starting HD and 1,075 starting PD (figure 12.1, table 12.3). UKRR 2015 incident

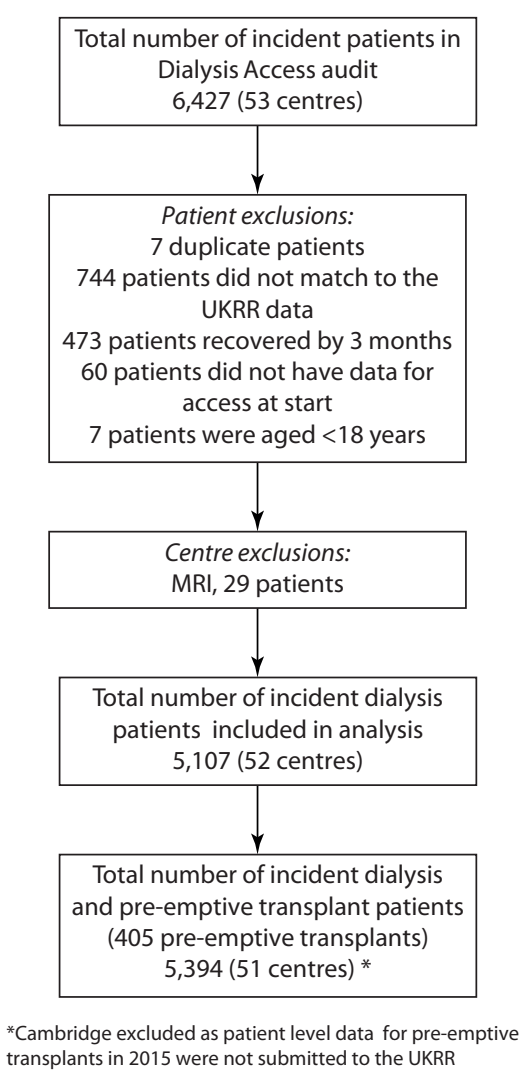

Fig. 12.1. STROBE flow diagram of patients included in the 2015 Multisite Dialysis Access audit 
Table 12.3. Demographics and characteristics of patients in the 2015 Multisite Dialysis Access audit, stratified by first dialysis access type

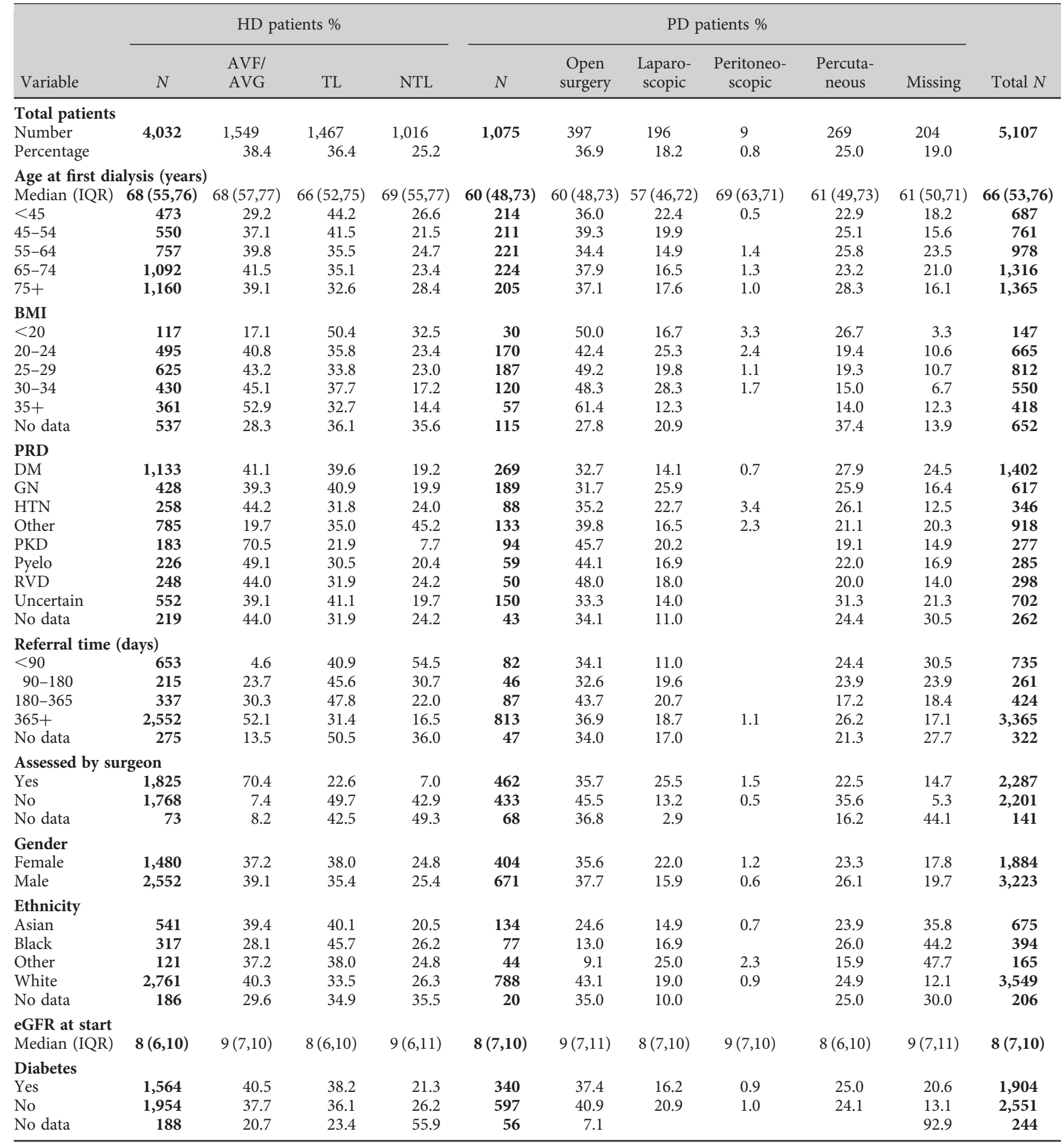

Centres with $>50 \%$ missing data for a variable were excluded from summary data and analyses relating to that variable, hence the total number of patients does not always sum to the total

IQR - interquartile range; BMI - body mass index; PRD - primary renal diagnosis; DM - diabetes mellitus; GN - glomerulonephritis; HTN - hypertension; PKD - polycystic kidney disease; Pyelo - pyelonephritis; RVD - renal vascular disease; HD - haemodialysis; PD - peritoneal dialysis; eGFR - estimated glomerular filtration rate; AVF - arteriovenous fistula; AVG - arteriovenous graft; TL - tunnelled line; NTL - non-tunnelled line 
data for centres submitting data were 3,968 HD and 1,076 PD patients. The slight over-reporting represents the inability to check all patients against the UKRR dataset, because some centres did not provide patient-level data. It is also possible that a small number of patients with AKI remained in the audit data on account of incomplete data at three months. Furthermore, it is possible that some patients who were excluded because they did not match to the UKRR database did not have AKI, but instead started dialysis towards the end of 2015 and the UKRR has not yet received data from renal centres.

\section{Data completeness}

Data completeness varied between 100\% (date of birth, gender, dialysis start date, first dialysis access and first dialysis modality) and $27.3 \%$ (date of access failure). The data on diabetes were supplemented by triangulation with UKRR comorbidity and primary renal diagnosis (PRD), increasing completeness of diabetic status data from $78.2 \%$ to $89.9 \%$. Of 51 centres that reported data on PD patients in $2014(N=1,069), 43$ completed the one year follow-up, returning data on 834 (78.0\%) patients. In these patients, 487 (58.4\%) were still on PD at one year with $76.8 \%$ of these $(374 / 487)$ still on their first catheter.

\section{Variations in first dialysis access}

The following observations can be made of incident dialysis access. These represent associations and do not imply causality. Data were unadjusted for patient factors.

- $51.4 \%$ of dialysis patients started therapy using an AVF/AVG or a PD catheter.

- 38.4\% of HD patients started therapy using an AVF or AVG.

- AVF use increased with increasing referral time, with corresponding reductions in TL/NTL use: 45.2\% of incident HD patients known to a nephrologist for over 90 days had an AVF/AVG which was below the Renal Association Audit standard of $60 \%$ (table 12.1).

- AVF use increased with increasing age and BMI, with corresponding reductions in TL/NTL use.

- AVG use was uncommon; used in only $0.9 \%$ of incident dialysis patients.
- Percutaneous PD catheter placement was less common with increasing BMI.

- Use of definitive access was high (80.5\%) for patients with polycystic kidney disease listed as their PRD (AVF 45.9\%; AVG 0.7\%; PD 33.9\%). There were corresponding low rates of TL/NTL use. For patients with 'other' listed as their PRD, AVF use was particularly low (16.9\%).

- Incident HD patients who had been reviewed by a surgeon at least three months prior to starting dialysis had higher AVF/AVG (70.4\% vs 7.4\%) and lower TL/NTL use $(29.6 \%$ vs $92.6 \%)$ than those who had not.

- Black patients starting HD had lower rates of AVF/ AVG use (28.1\%) than average (38.4\%).

Figures 12.2-12.7 assist interpretation of table 12.3 by including annual transplant data. Transplant data were included to provide a more complete depiction of incident RRT patterns. Data remained otherwise unadjusted. For a more detailed analysis of transplantation, see chapters 3 and 11 of this Annual Report. Data were plotted and stratified by age (figure 12.2), BMI (figure 12.3), PRD (figure 12.4), referral time (figure 12.5), diabetic status (figure 12.6) and surgical referral (figure 12.7). Centres with $>50 \%$ missing data for a variable were excluded, as detailed in the figure legend. BMI data on PTx recipients are not presented due to low data returns, although it is recognised that very few transplant recipients will have BMI $>35$. Transplant data were not presented against surgical referral data because all patients who received a PTx will have received surgical review. HD and PD data are displayed separately in figure 12.7 because the surgical pathways for vascular and PD access differ. Late presenting patients were excluded from this analysis. The following observations can be made:

- Rising use of AVF/AVG with increasing age was associated with falling rates of transplant and PD.

- Amongst incident RRT patients with BMI <20, PD use was low (20.4\%) and TL/NTL use was high (66.0\%). Otherwise the rising use of AVF/AVG with increasing BMI was associated with falling rates of $\mathrm{PD}$.

- PRD had a variable association with use of definitive dialysis access and PTx. For example, for polycystic kidney disease both definitive dialysis access (60.4\%) and PTx (24.3\%) were common. Where PRD was listed as 'other', definitive dialysis access (29.7\%) 

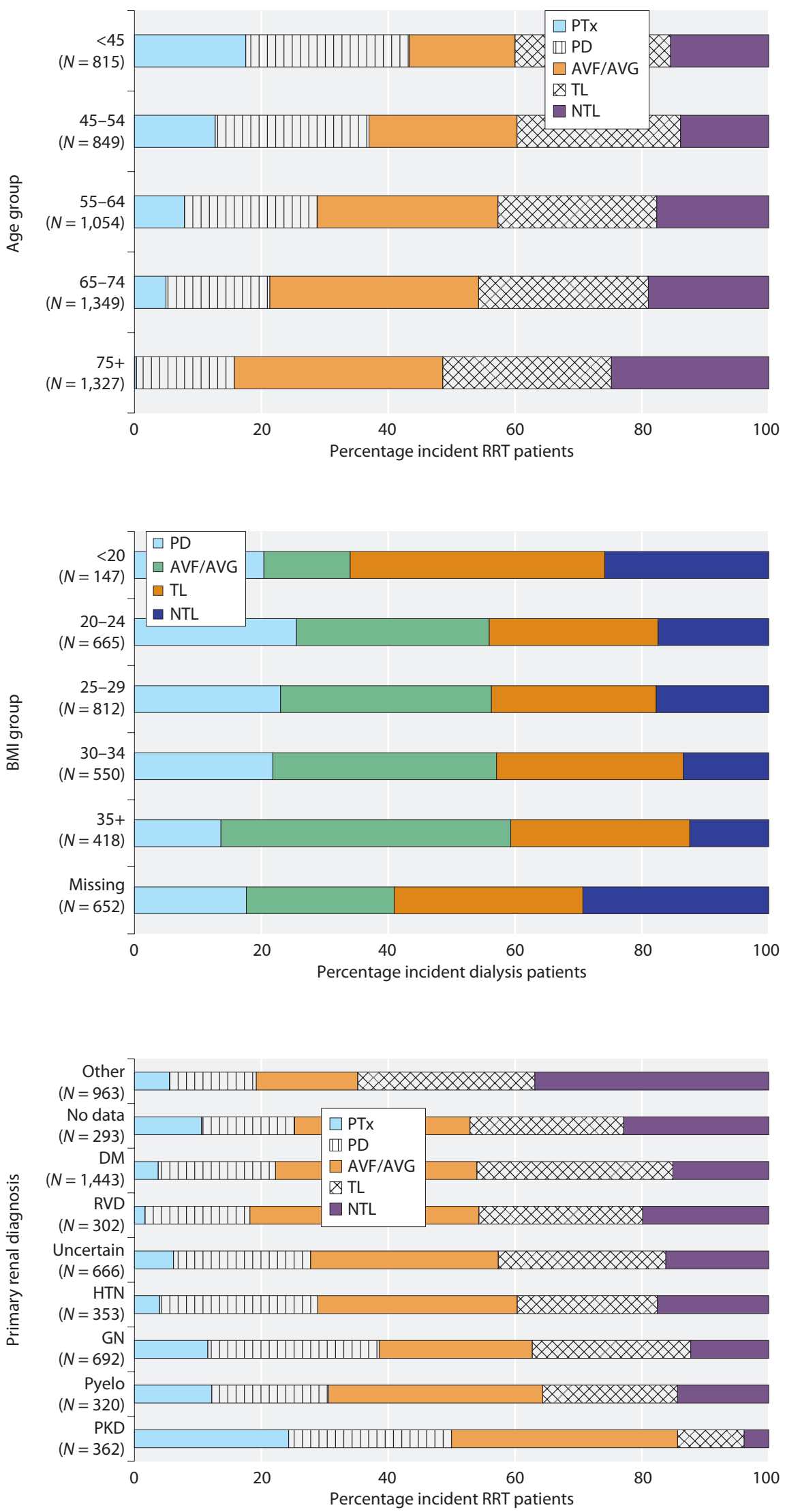

Fig. 12.2. Percentage of incident RRT patients by age group, 2015 Number of patients in each group in brackets. PTx - pre-emptive transplant; PD - peritoneal dialysis; AVF - arteriovenous fistula; AVG - arteriovenous graft; TL - tunnelled line; NTL - non-tunnelled line

Fig. 12.3. Percentage of incident dialysis patients by BMI group, 2015

Number of patients in each group in brackets. 15 centres were excluded due to $>50 \%$ missing BMI data.

PD - peritoneal dialysis; AVF - arteriovenous fistula; AVG - arteriovenous graft; TL - tunnelled line; NTL - non-tunnelled line; BMI - body mass index

Fig. 12.4. Percentage of incident RRT patients by primary renal diagnosis, 2015 Number of patients in each group in brackets. PRD groups are sorted by decreasing proportion of patients initiating RRT with a HD catheter. PRD - primary renal diagnosis; DM - diabetes mellitus; GN - glomerulonephritis; HTN hypertension; PKD - polycystic kidney disease; Pyelo - pyelonephritis; RVD - renal vascular disease

PTx - pre-emptive transplant; PD - peritoneal dialysis; AVF - arteriovenous fistula; AVG - arteriovenous graft; TL - tunnelled line; NTL - non-tunnelled line 

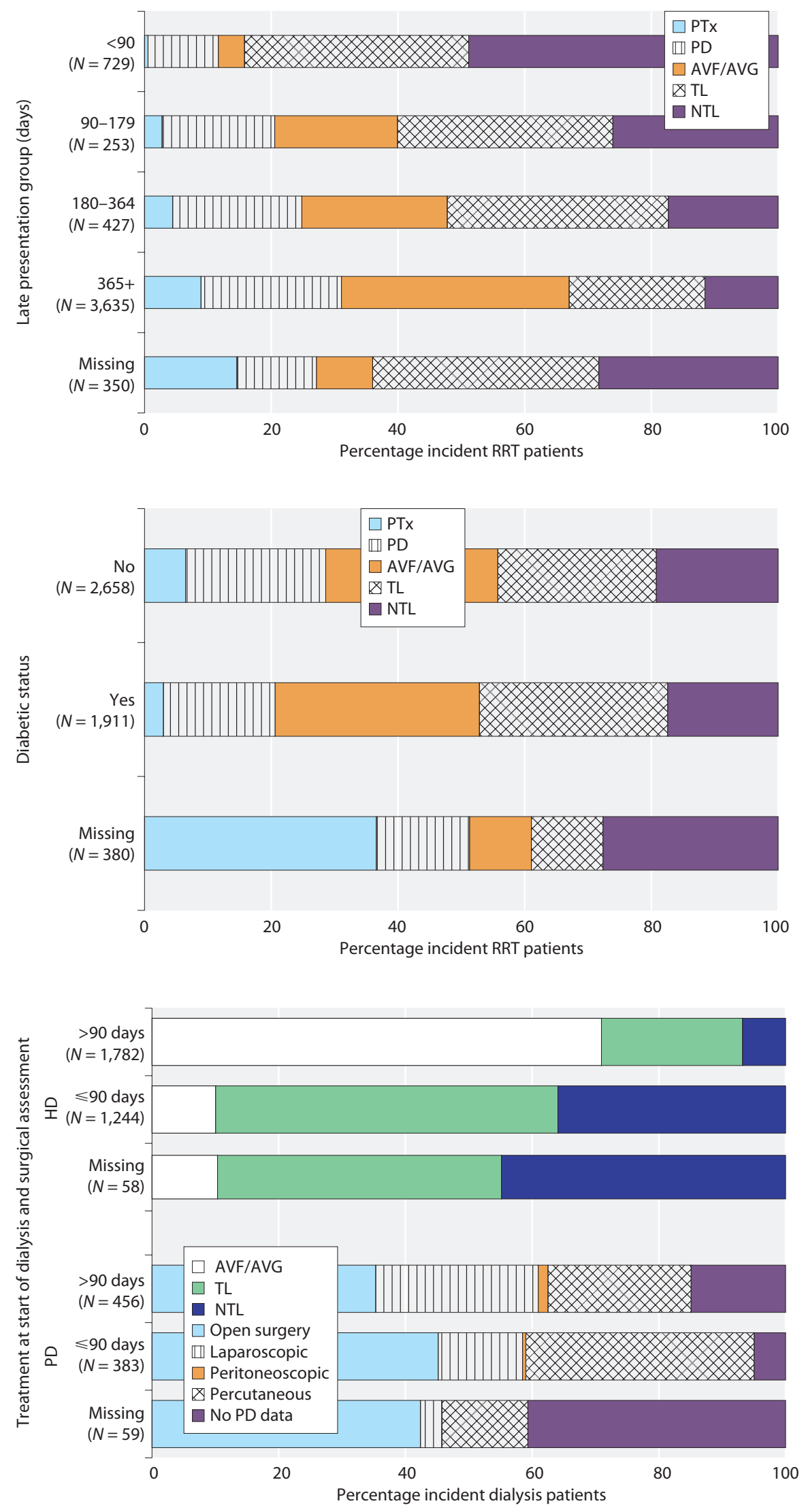

Fig. 12.5. Percentage of incident RRT patients by late presentation group, 2015 Number of patients in each group in brackets. PTx - pre-emptive transplant; PD - peritoneal dialysis; AVF - arteriovenous fistula; AVG - arteriovenous graft; TL - tunnelled line; NTL - non-tunnelled line

Fig. 12.6. Percentage of incident RRT patients by diabetic status, 2015

Number of patients in each group in brackets. Two centres were excluded due to $>50 \%$ missing diabetes data after triangulation with UKRR data PTx - pre-emptive transplant; PD - peritoneal dialysis; AVF - arteriovenous fistula; AVG - arteriovenous graft; TL - tunnelled line; NTL - non-tunnelled line

Fig. 12.7. Percentage of incident dialysis patients stratified by assessment by a surgeon within 3 months before starting RRT and access at start of dialysis, 2015 Number of patients in each group in brackets. Late presenting patients were excluded from the analysis

AVF - arteriovenous fistula; AVG -

arteriovenous graft; TL - tunnelled line; NTL non-tunnelled line; PD - peritoneal dialysis

Hole/Caskey/Evans/Fluck/Kumwenda/ Steenkamp/Wilkie 
and PTx (5.5\%) were both uncommon. In renovascular disease definitive dialysis access was established in 52.6\% of incident patients, whilst PTx was very rare $(1.7 \%)$.

- Increasing referral time was associated with a gradual increase in AVF/AVG, PD and PTx use, with corresponding reductions in use of TL/NTL. This pattern continued as referral time increased beyond 365 days.

- $63.5 \%$ of incident RRT patients known to a nephrologist for over 90 days had definitive access or a transplant. Whilst the Renal Association present this as an audit standard, no minimum standard is set (table 12.1).

- PD was initiated for only $11.1 \%$ of late presentations.

- Patients with diabetes were more likely to use an AVF/AVG and less likely to receive PTx or PD than patients without diabetes.

- AVF/AVG use was much higher amongst haemodialysis recipients referred to a surgeon $>90$ days before dialysis initiation $(70.9 \%)$ than those who were not $(10.0 \%)$.

\section{Variations in first dialysis access by renal centre}

Figure 12.8 plots incident RRT first access method stratified by centre. Practice variation was apparent. Initiating $\mathrm{HD}$ via an $\mathrm{AVF} / \mathrm{AVG}$ ranged between $<15 \%$ (London West, Carlisle) and $>40 \%$ (Cardiff, Sheffield, Gloucester, York, Colchester). Initiating HD via a TL ranged between $<5 \%$ (Belfast) and $>40 \%$ (London West, Colchester, West NI). Initiating with a PD catheter ranged between 0\% (Clwyd, Colchester, Plymouth) and $>40 \%$ (Derby, Newry, Carlisle). There does not seem to be a relationship between the rate of definitive access use and whether a centre is a transplant or nontransplanting centre.

Table 12.4 provides centre-level data for incident dialysis access, grouping patients by time of presentation to nephrology (early $\geqslant 90$ or late $<90$ days before initiating dialysis). Late presentation was associated with low rates of definitive access placement (15.3\%). Peritoneal catheter placement accounted for $73.2 \%$ of definitive access placed in late presenting patients. Sixteen centres had no late presenting patients dialysing with definitive access at initiation. Some centres were able to establish definitive vascular access for late presenting patients, although absolute numbers of patients were small. Surgical referral was made 90 days or more before dialysis initiation for $51.2 \%$ of incident patients, and ranged between $>90 \%$ (London Barts, Middlesbrough) and $<20 \%$ (Plymouth, Southend).

Table 12.5 provides centre-level data for dialysis access three months after initiation, grouping patients by time of initial presentation to nephrology (early $\geqslant 90$ or late $<90$ days before initiating dialysis). Late presentation remained associated with low rates of definitive access use at three months (15.2\%) compared with early presentation $(60.0 \%)$. TL was the mode of access for $62.4 \%$ of late presenting patients at three months. Definitive access was similar at initiation and three months later for late presenters and early presenters. Of early presenters, $1.8 \%$ were transplanted by three months with an overall fall in use of NTLs amongst this group. Of late presenting patients, $0.1 \%$ were transplanted by three months. Sixteen centres had no late presenting patients dialysing with definitive access at three months. A small number of centres were able to establish definitive access in at least $40 \%$ of late presenting patients by three months (Derby, London St George's, Cardiff).

Table 12.6 shows dialysis access three months after initiation, stratified by first access type. The shaded cells highlight proportions of patients who continued to use their initial dialysis access at three months. Of patients who initiated dialysis with definitive access, $86.2 \%$ continued with the same access at three months and 88.2\% had definitive access or a transplant, whilst 5.9\% converted to TL/NTL. Of patients who started dialysis without definitive access, $12.8 \%$ received a transplant or were dialysing with definitive access at three months. Of patients who initiated dialysis with a TL, 78.9\% continued with a TL at three months and only $12.9 \%$ had converted to definitive access or a transplant. Death before three months was much more common in patients initiating dialysis with a NTL than with any other form of initial access (22.5\%). Of those patients who initiated dialysis with a NTL and survived to three months, $78.3 \%$ converted to a TL.

Figure 12.9 provides a funnel plot of the percentage of patients starting HD with an AVF or AVG. Late presenting patients were excluded as a surrogate for 'unplanned dialysis initiation' as per the Renal Association guidelines (table 12.1). This analysis shows that the majority of UK renal centres fell below the Renal Association audit standard of $\geqslant 60 \%$ AVF/AVG use at 'planned' HD initiation. Only ten centres achieved the target. All these centres had $<65$ incident HD patients, although 


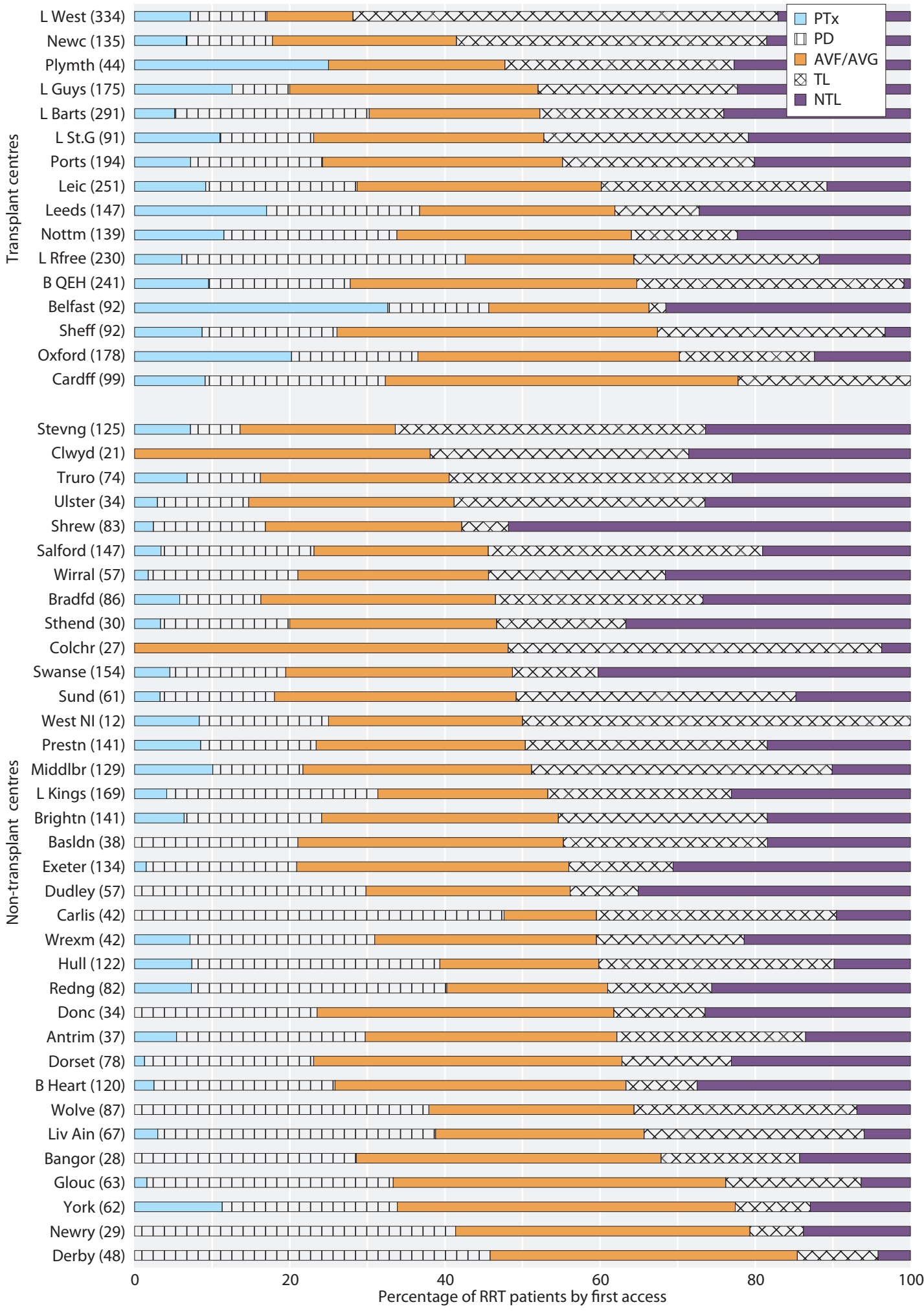

Fig. 12.8. Incident RRT first access method for patients in the 2015 Multisite Dialysis Access audit, stratified by renal centre Centre size (patient numbers) in brackets. Centres are stratified by transplanting/non-transplanting centre and sorted by proportion of patients initiating RRT with a HD catheter (TL/NTL). PTx - pre-emptive transplant; PD - peritoneal dialysis; AVF - arteriovenous fistula; AVG - arteriovenous graft; TL - tunnelled line; NTL - non-tunnelled line; RRT - renal replacement therapy 
Table 12.4. Modality at start of dialysis and access in use for patients in the 2015 Multisite Dialysis Access audit, by early and late presentation at dialysis initiation, by centre, including surgical referral rates within three months before start of dialysis

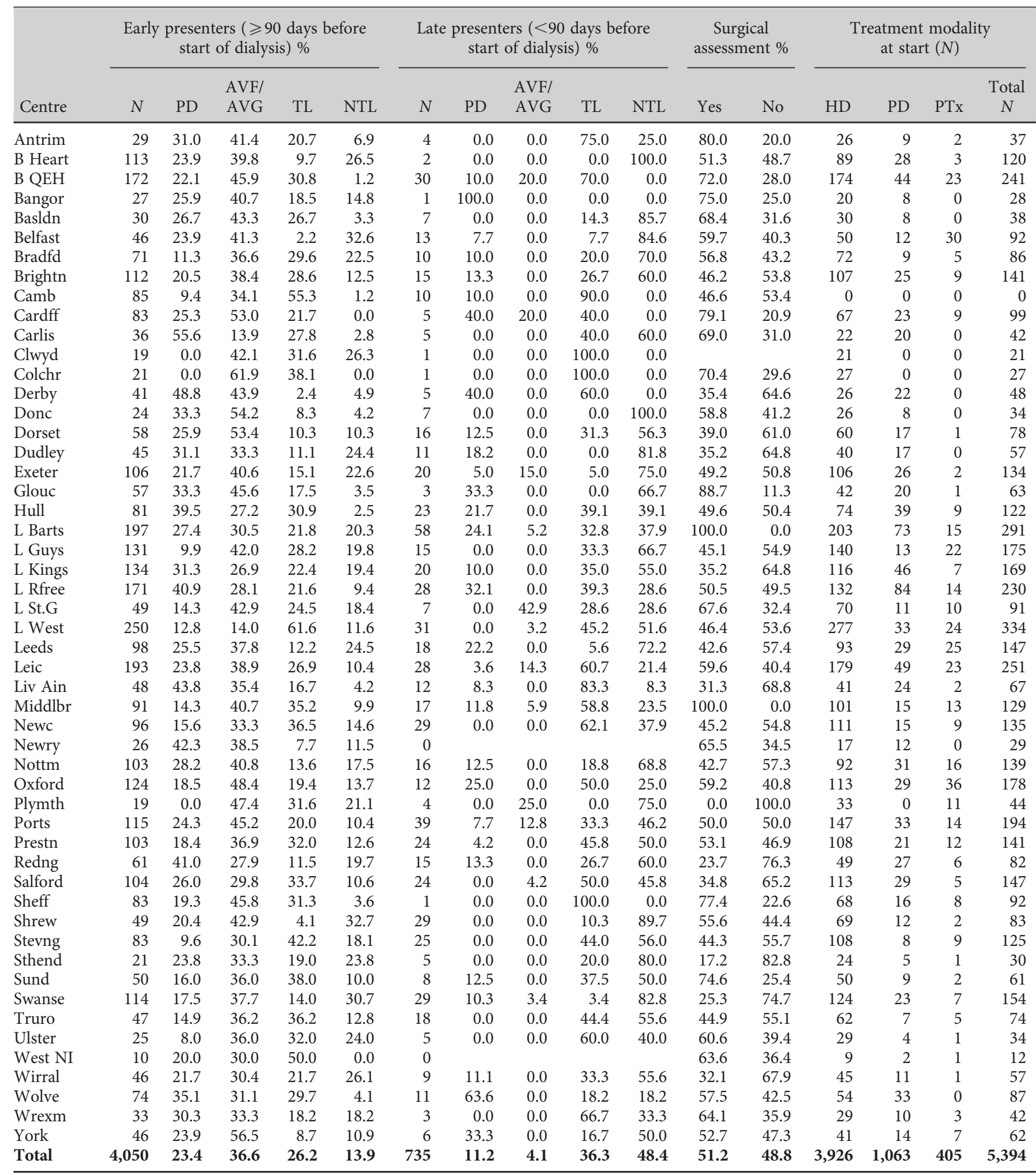

PTx - pre-emptive transplant; HD - haemodialysis; PD - peritoneal dialysis; AVF - arteriovenous fistula; AVG - arteriovenous graft; TL - tunnelled line; NTL - non-tunnelled line 
Table 12.5. Modality at three months after start of dialysis and access in use for patients in the 2015 Multisite Dialysis Access audit, by early and late presentation at dialysis initiation, by centre

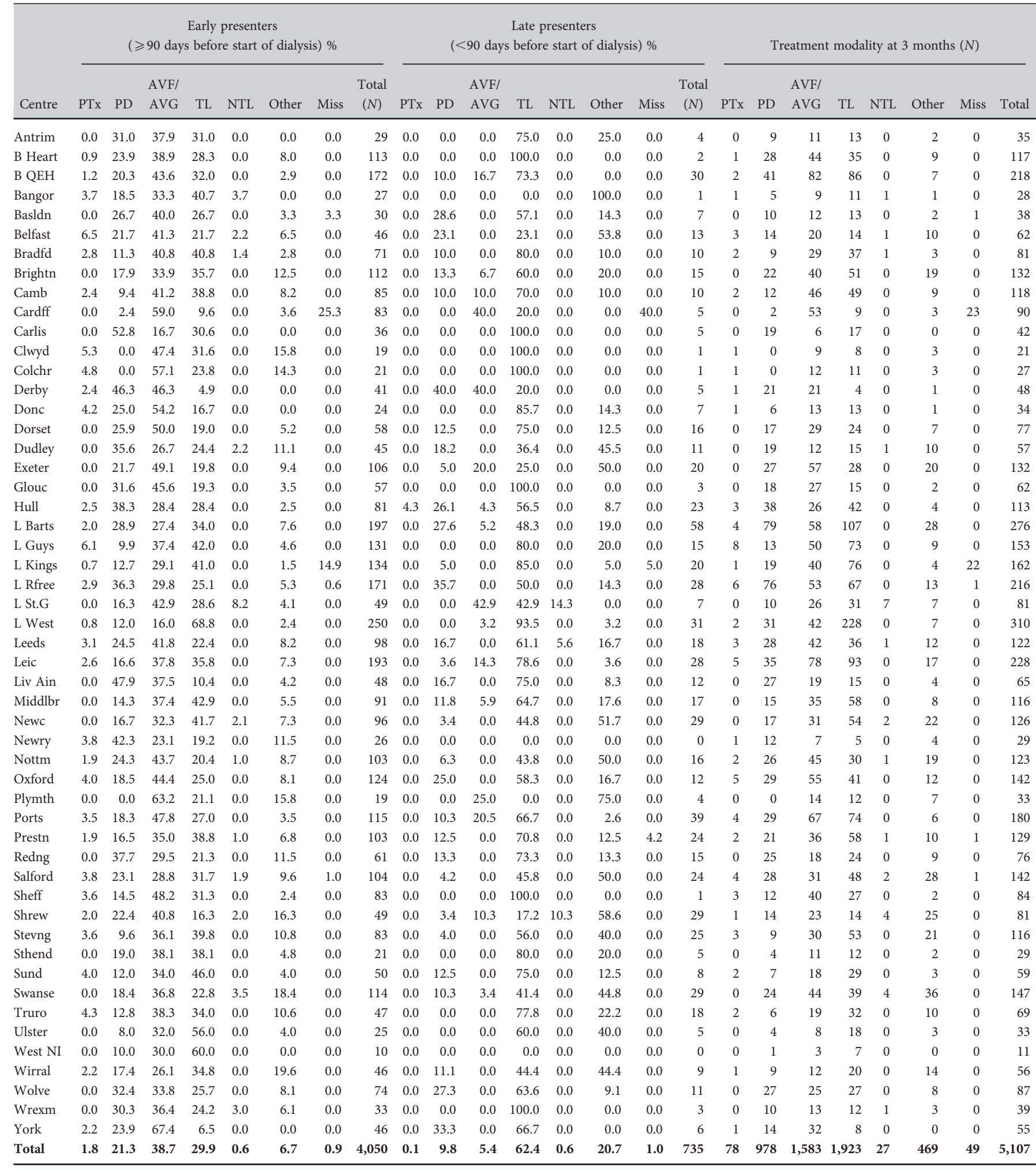

PTx - pre-emptive transplant; PD - peritoneal dialysis; AVF - arteriovenous fistula; AVG - arteriovenous graft; TL - tunnelled line; NTL - non-tunnelled line; Miss - missing data 
Table 12.6. Dialysis access at three months since dialysis start for patients in the 2015 Multisite Dialysis Access audit, stratified by first access used

\begin{tabular}{lcccccccc}
\hline & \multicolumn{7}{c}{ Access in use at three months (\%) } \\
\cline { 2 - 8 } $\begin{array}{l}\text { Access in use at } \\
\text { first dialysis }(N)\end{array}$ & AVF/AVG & TL & NTL & PD catheter & Transplanted & Died & Stopped/LTFU & No data \\
\hline AVF/AVG (1,549) & 88.4 & 5.1 & 0.1 & 0.1 & 1.5 & 2.9 & 1.9 & 0.0 \\
TL $(1,467)$ & 9.3 & 78.9 & 0.3 & 2.2 & 1.4 & 6.8 & 1.0 & 0.1 \\
NTL (1,016) & 7.0 & 60.6 & 1.9 & 5.2 & 0.5 & 22.5 & 1.8 & 0.5 \\
PD catheter (1,075) & 0.5 & 6.6 & 0.2 & 83.0 & 2.8 & 2.4 & 0.7 & 3.9 \\
\hline
\end{tabular}

Shaded cells highlight the percentage of patients who remained on the same modality at three months PD - peritoneal dialysis; AVF - arteriovenous fistula; AVG - arteriovenous graft; TL - tunnelled line; NTL - non-tunnelled line; LTFU lost to follow-up

the majority of centres of this size failed to meet the audit standard.

Figure 12.10 depicts the percentage of incident HD patients by first access used, stratified by time between date of first access formation attempt and HD initiation. Data from patients incident to dialysis in 2014 and 2015 are included. Date of first access was collected for the first time in 2014 and has not previously been presented in analyses in this chapter. Longer duration between first attempt at forming dialysis access and first HD session was associated with greater levels of AVF/AVG use at initiation. Amongst patients for whom the first attempt at forming dialysis access was made more than one year before starting $\mathrm{HD}, 89.0 \%$ initiated with AVF/ AVG; whereas for those patients for whom the first attempt at forming dialysis access was made $<90$ days before starting dialysis, $15.6 \%$ commenced HD with an AVF/AVG. The biggest increment in definitive dialysis

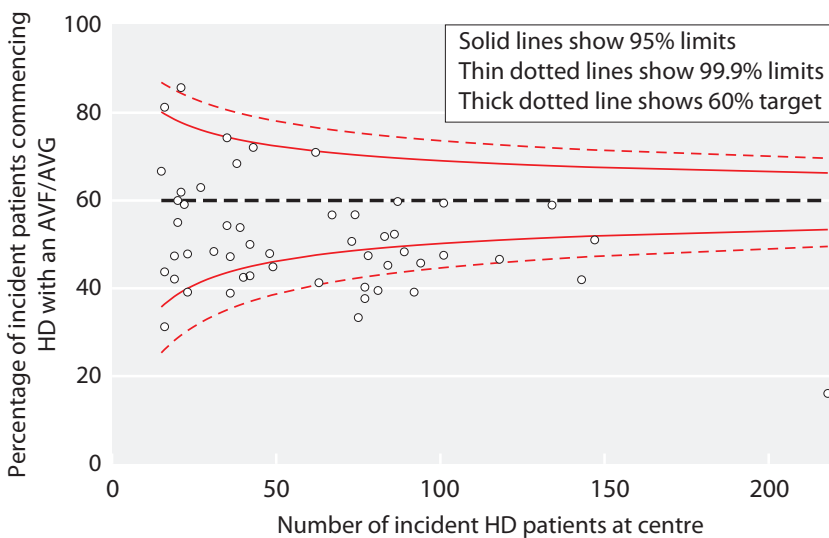

Fig. 12.9. Funnel plot of the percentage of $\mathrm{HD}$ patients in the 2015 Multisite Dialysis Access audit who commenced dialysis with an AVF/AVG

Patients who were first seen by a nephrologist $<90$ days from initiating dialysis were excluded. Centres with $<10$ patients receiving HD were excluded. HD - haemodialysis; AVF - arteriovenous fistula; AVG - arteriovenous graft

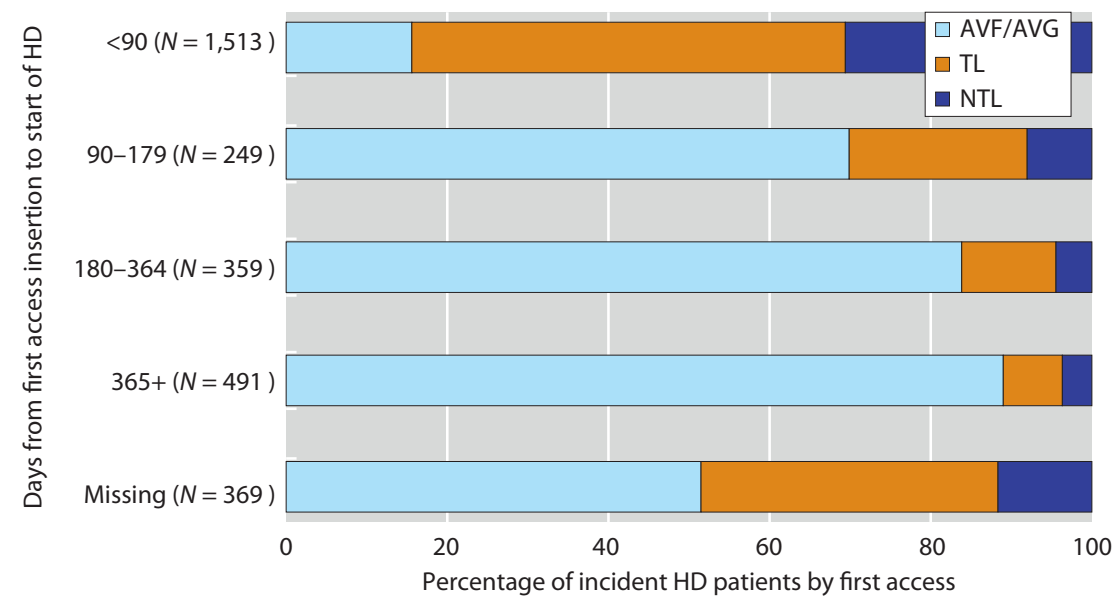

Fig. 12.10. Percentage of incident HD patients by first access used in the 2014 and 2015 Multisite Dialysis Access audits stratified categorically by days $(<90 ; 90-179 ; 180-364$; $365+$ ) from first access attempt

Number of patients in each category in brackets. Latepresenting patients were excluded from this analysis. Three centres were excluded due to $>50 \%$ missing data for date of first access attempt. HD -

haemodialysis; AVF - arteriovenous fistula; AVG arteriovenous graft; TL - tunnelled line; NTL - nontunnelled line; Miss - missing data 
access occurred between $<90$ and $\geqslant 90$ days. Three centres were excluded due to $>50 \%$ missing data for date of first access attempt. Furthermore, the data field did not specify which access was attempted, so it cannot be assumed that first access attempt and access used on first session were the same. Missing data had a similar distribution of access use to those seen in patients for whom data were provided, suggesting no systematic tendency for early or late presenting patients to be more or less likely to have missing data.

\section{Variations in prevalent dialysis access by renal centre}

Figure 12.11 provides a funnel plot of the percentage of prevalent dialysis patients receiving $\mathrm{PD}$ or $\mathrm{HD}$ via an AVF/AVG. Seventeen centres met the Renal Association audit standard of $\geqslant 80 \%$ for definitive access use (thick dotted line). Thirteen centre-level exclusions were made for this analysis due to non-completion of prevalent dialysis access data and $>10 \%$ differences between centre-reported and UKRR numbers of patients receiving dialysis.

Figure 12.12 depicts dialysis access for prevalent patients by centre. Wide practice variation was apparent. Rates of definitive access ranged between $>90 \%$ (Derby, Birmingham Heartlands, Dorset) and $<50 \%$ (London West, Ulster). PD accounted for between $>25 \%$ (Dudley,

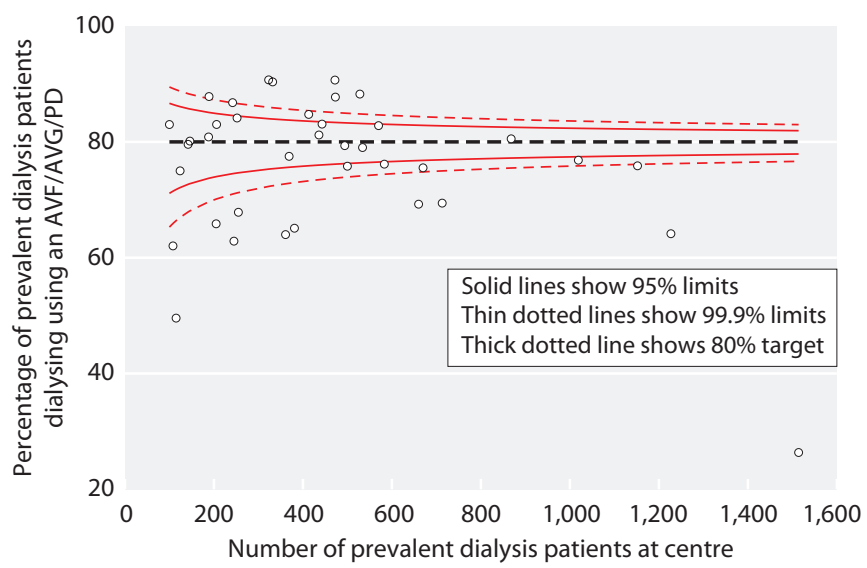

Fig. 12.11. Funnel plot of the percentage of prevalent patients in the 2015 Multisite Dialysis Access audit receiving PD or HD via $\mathrm{AVF} / \mathrm{AVG}$

A total of 13 centre-level exclusions were made for this analysis due to non-completion of prevalent dialysis access data and $>10 \%$ differences between centre-reported and UKRR numbers of patients receiving dialysis. HD - haemodialysis; PD - peritoneal dialysis; AVF - arteriovenous fistula; AVG - arteriovenous graft
Carlisle) and $<5 \%$ (London West, London Guys) of prevalent definitive access use. Thirteen centre-level exclusions were made for this analysis due to non-completion of prevalent dialysis access data and $>10 \%$ differences between centre-reported and UKRR numbers of patients receiving dialysis.

\section{Peritoneal dialysis audit one-year follow-up by renal centre}

Figure 12.13 shows RRT modality one year after commencing PD by centre. Data for this analysis came from the 2015 one year follow-up for patients incident to dialysis in 2014 . Centres with $100 \%$ missing data at one year, or fewer than five PD patients were excluded. The percentage of patients remaining on $\mathrm{PD}$ or who were transplanted one year after initiation ranges between $10 \%$ (Stevenage) and $>85 \%$ (Cambridge, Doncaster, Wrexham, Plymouth, Leeds, Salford) with an overall mean of $72.8 \%$. Of patients continuing dialysis (i.e. censoring for death, transplant and withdrawal), 76.6\% of patients starting PD continued to use this modality one year later.

Figure 12.14 depicts PD catheter insertion technique stratified by centre. The five centres reporting fewer than five patients on PD were not considered for analysis. Surgical techniques include open and laparoscopic. Nonsurgical techniques include percutaneous and peritoneoscopic insertion. There was considerable practice variation. Seventeen centres performed non-surgical PD catheter placement, accounting for $25.9 \%$ of all catheters placed and 13 of these centres placed $>50 \%$ of their PD catheters this way. Six placed $>90 \%$ of their PD catheters percutaneously (Southend, Gloucester, Derby, Birmingham Heartlands, Salford, Wolverhampton). At the 17 centres that place non-surgical PD catheters, $25.9 \%$ of incident RRT patients started PD, compared with $21.0 \%$ overall. Approximately $48 \%$ percent of incident RRT patients started PD at the six centres that placed $>90 \%$ of their catheters percutaneously.

Figure 12.15 displays PD catheter insertion technique by referral time. There does not appear to be a strong relationship between referral time and technique for PD catheter insertion. This suggests that the PD access referral pathway was less dependent on timely referral than the vascular access pathway.

Figure 12.16 presents the percentage of incident PD patients by catheter insertion technique and BMI 


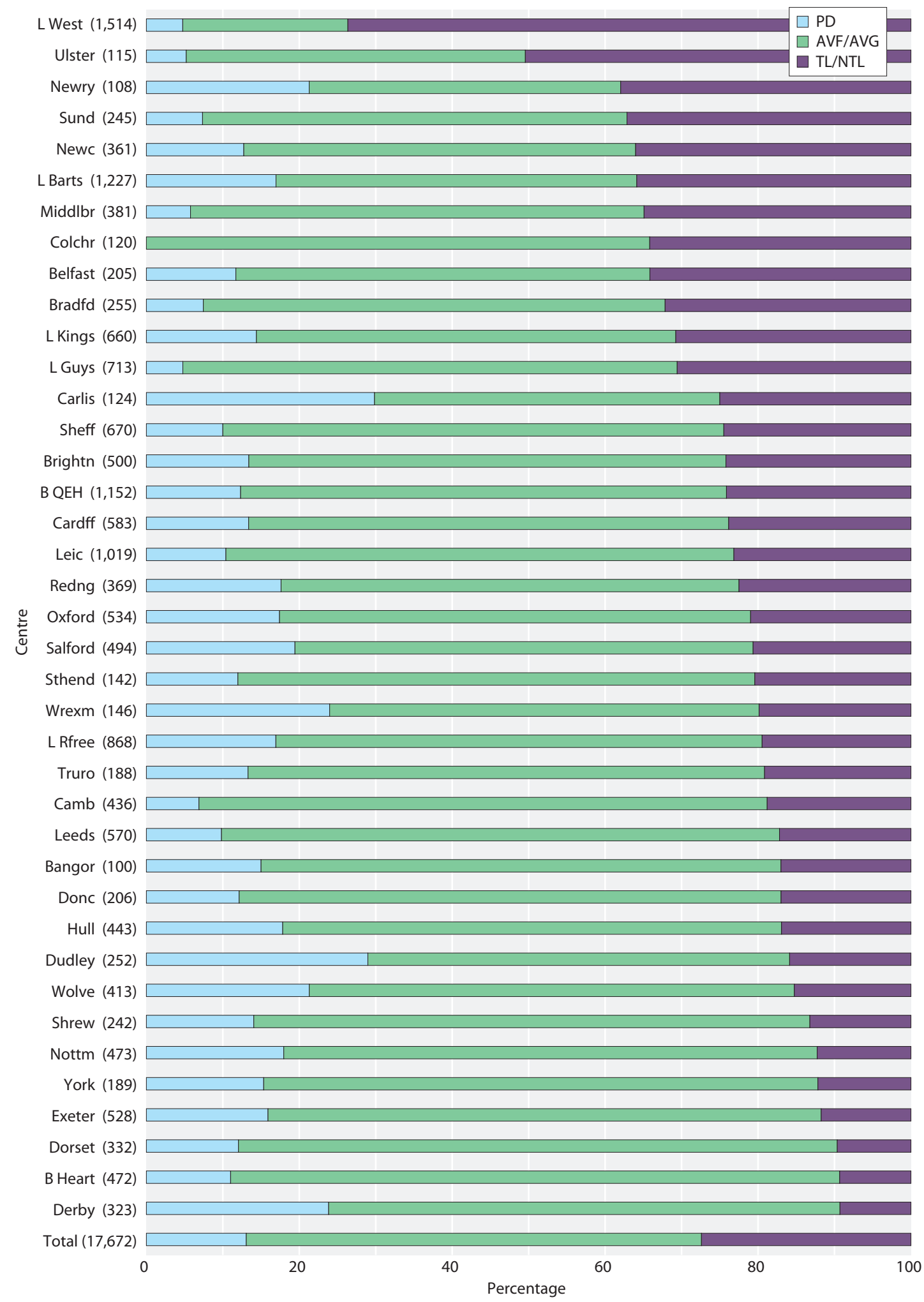

Fig. 12.12. Prevalent dialysis access by centre for patients in the 2015 Multisite Dialysis Access audit Centre size (patient numbers) in brackets. Centres are sorted by proportion of patients initiating RRT with a HD catheter. HD - haemodialysis; PD - peritoneal dialysis; AVF - arteriovenous fistula; AVG - arteriovenous graft; TL - tunnelled line; NTL - non-tunnelled line 


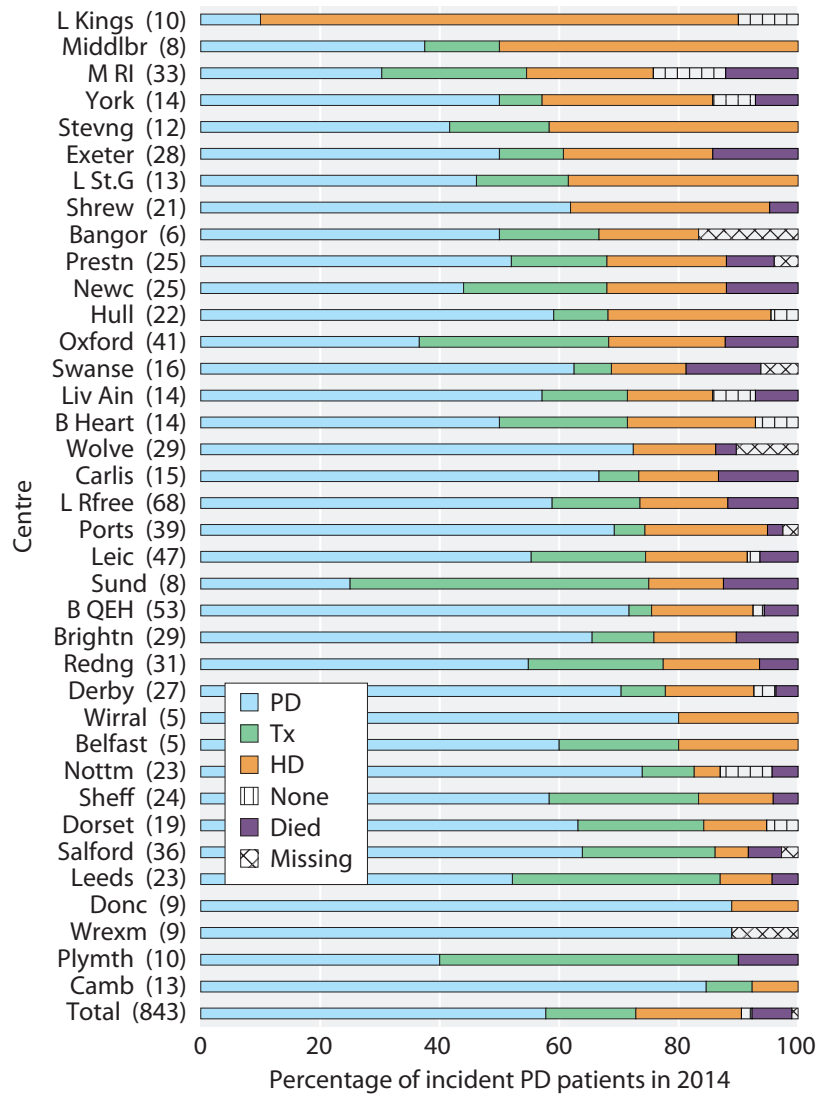

Fig. 12.13. Modality at one year after commencing PD in 2014, by centre

Number of patients receiving PD at each centre in brackets. Centres with $100 \%$ missing treatment data at one year or fewer than five PD patients were excluded. Centres are sorted by proportion of patients transplanted or remaining on PD. PD - peritoneal dialysis; HD - haemodialysis; Tx - transplanted

group. Associations between BMI and PD catheter insertion technique do not appear to be strong. An open surgical approach was used more frequently than any other technique (43.0\%). Laparoscopic surgery was used less in patients with a BMI >35 (11.7\%) and was compensated by an increase in the use of open surgery (61.7\%). Rates of laparoscopic insertion were also low amongst individuals with $\mathrm{BMI}<20(16.1 \%)$. This was compensated for by greater use of open $(54.8 \%)$ and percutaneous approaches (25.8\%).
Figure 12.17 shows a funnel plot of the percentage of PD catheter failures within one year of initiating dialysis. Data are from the one year PD follow-up audit of patients incident to PD in 2014. PD catheter failure was censored for transplantation, elective transfer to HD or death. Of the 30 centres for which data were available, none were above the $95 \%$ limit for PD catheter failure. However, four centres were below the lower $99.9 \%$ limit, none of which reported a failed PD catheter. The average one year catheter failure rate of $13.3 \%$ is an improvement on that which was reported in previous years $(20.2 \%$ in 2014). Nine centres reported peritonitis within two weeks of $\mathrm{PD}$ catheter insertion, with rates ranging between $1.3 \%$ and $13.0 \%$ of inserted catheters. Twentynine centres reported no cases of peritonitis within two weeks. These results should be interpreted with caution due to missing data and small numbers of patients in some centres.

Figure 12.18 shows comparative access failure by access type within three months of initiating dialysis. Data were drawn from the 2014 and 2015 Multisite Dialysis Access audits. Access failure was defined as a documented date of failure/discontinuation recorded within three months of starting dialysis, unless a centre comment indicated that it was a planned discontinuation. Failure rates appeared higher for PD than for HD access. Numbers of AVGs and peritoneoscopically inserted PD tubes were very low, hence the wide confidence intervals (CIs) for these data. There was no signal from these data to suggest that sub-types of HD or PD access were more or less likely to fail at three months.

Figure 12.19 shows causes of PD catheter access failure within one year of initiating dialysis in 112 catheters reported from the one year PD follow-up audit of patients incident to dialysis in 2014. Infection was a more frequent cause of failure for percutaneously inserted than surgically placed PD catheters and for open compared with laparoscopic insertion. No leaks or hernias were reported for percutaneously inserted or failures reported in peritoneoscopically inserted PD catheters. The relatively small number in this analysis increases the likelihood that differences in causes of failure between subgroups are due to chance. 


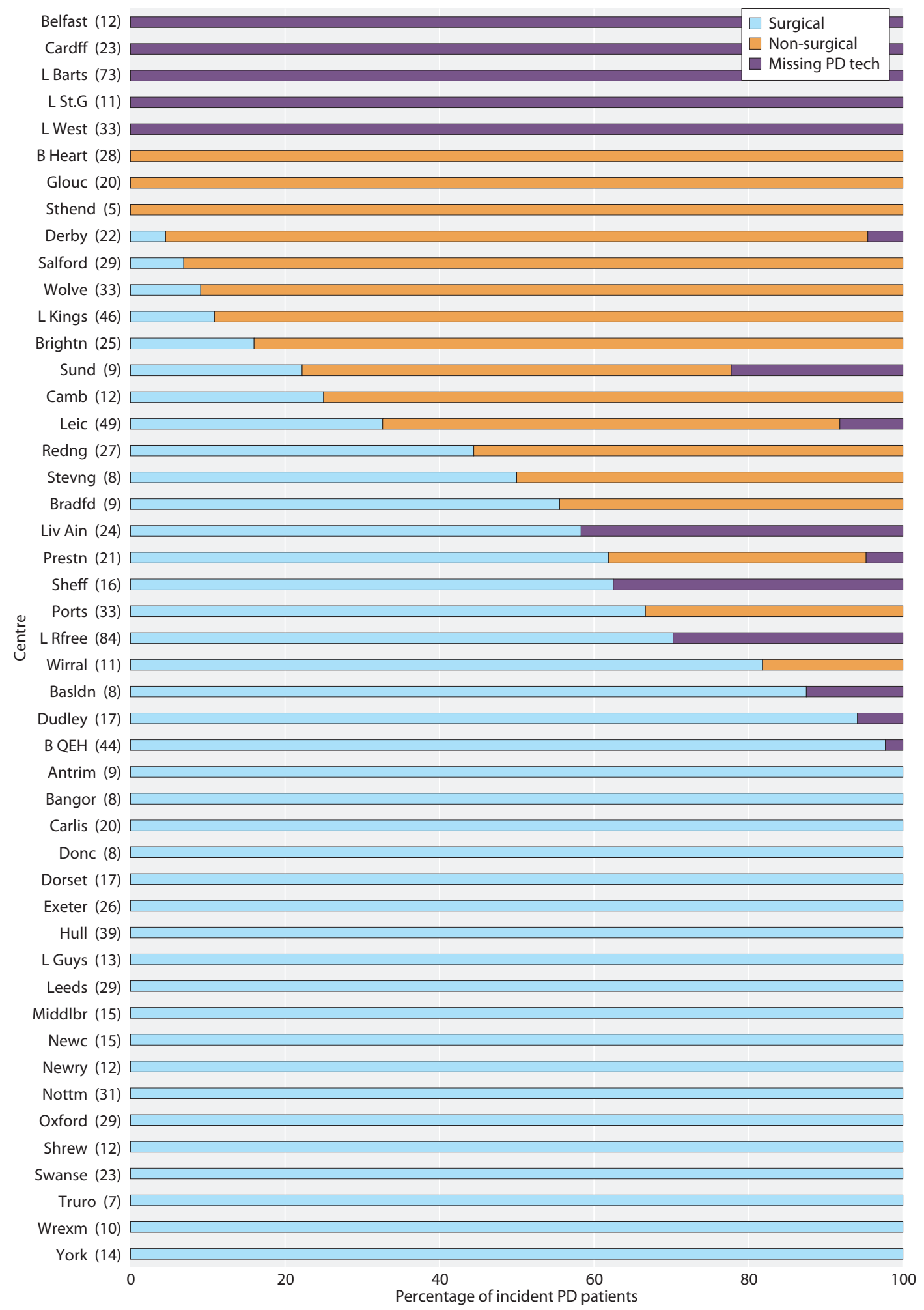

Fig. 12.14. PD catheter insertion technique (surgical vs non-surgical) stratified by centre for patients in the 2015 Multisite Dialysis Access audit

Number of patients receiving PD at each centre in brackets. Centres are sorted by proportion of catheters inserted by surgical technique. Centres reporting fewer than five patients on PD were excluded from this analysis. Due to small numbers in the subcategories of surgical insertion techniques, open and laparoscopic insertions are grouped as 'surgical'; peritoneoscopic and percutaneous as 'non-surgical'. PD - peritoneal dialysis 

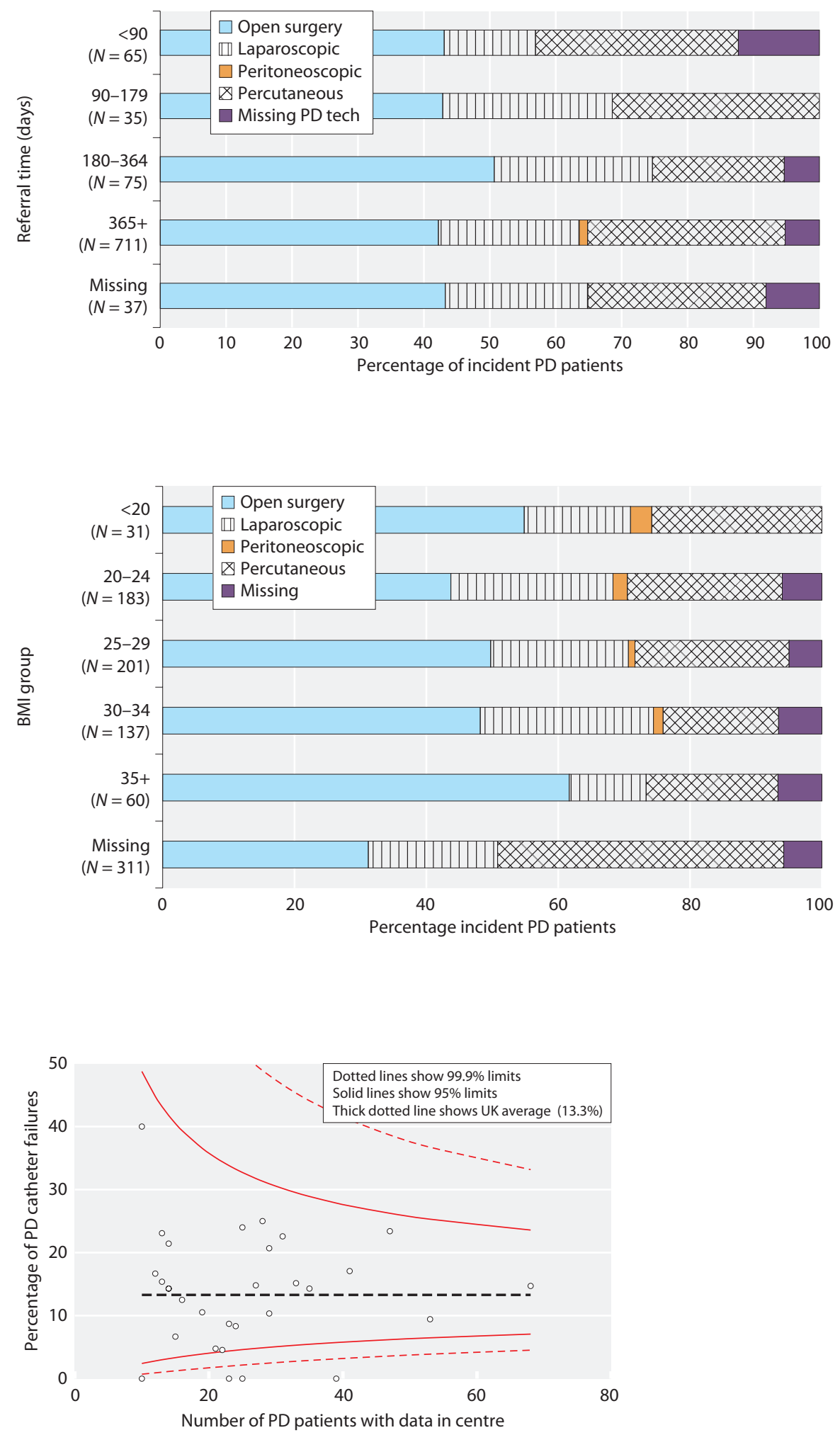

Fig. 12.17. Funnel plot of the percentage of PD catheter failures within one year of start date for patients incident to PD in 2014 Thirteen centres with $<10$ patients on PD were excluded from this analysis, along with eight centres that did not return data for the one year follow-up. PD - peritoneal dialysis
Fig. 12.15. PD catheter insertion technique by referral time (days) for patients in the 2015 Multisite Dialysis Access audit

Number of patients in each category in brackets. Referral time was measured between first nephrology input (inpatient/outpatient) and initiating dialysis. Five centres were excluded from this analysis due to $>50 \%$ missing data for PD catheter insertion technique. PD - peritoneal dialysis

Fig. 12.16. Percentage of incident $P D$ patients by catheter insertion technique and BMI group for patients in the 2015 Multisite Dialysis Access audit Number of patients in each category in brackets. Five centres were excluded from this analysis due to $>50 \%$ missing data for PD catheter insertion technique and 15 centres due to $>50 \%$ of missing data for BMI. $\mathrm{PD}=$ peritoneal dialysis; $\mathrm{BMI}=$ body mass index 

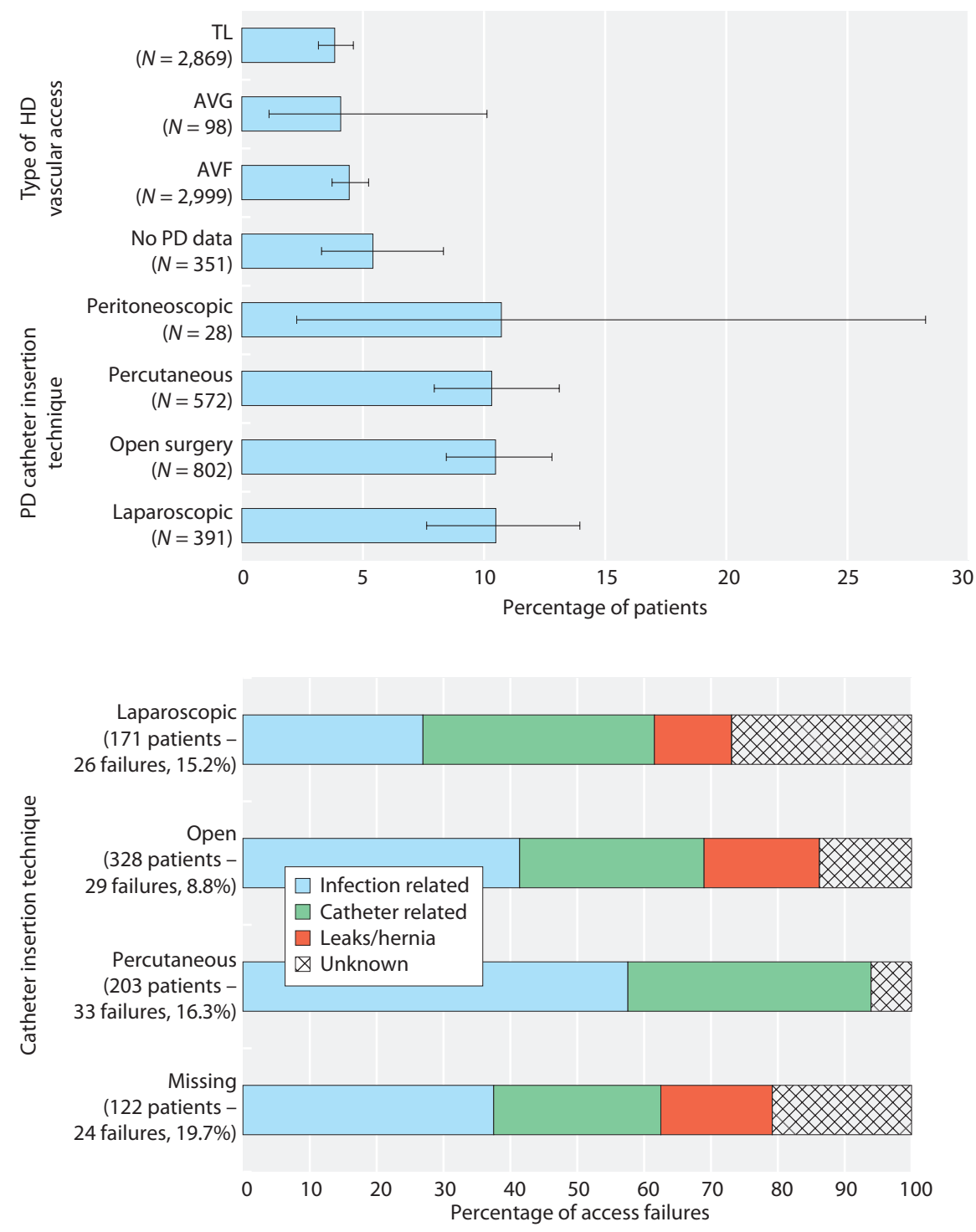

Fig. 12.18. Percentage of patients experiencing failure of first access within three months, by type of first access, for patients in the 2014 and 2015 Multisite Dialysis Access audits PD - peritoneal dialysis; AVF - arteriovenous fistula; AVG - arteriovenous graft; TL - tunnelled line
Fig. 12.19. Percentage of $\mathrm{PD}$ catheter access failures within one year of starting dialysis, from PD follow-up data, 2015 $\mathrm{PD}$ - peritoneal dialysis

\section{Part 2 - Accumulated results from the 2011- 2015 annual Multisite Dialysis Access audits}

\section{Data completeness}

Over the five years since the multisite access audit was initiated, data on a total of 23,639 incident dialysis patients have been collected. The UKRR holds data for 33,034 incident dialysis patients over this period with patient-level data on dialysis access being available for $71.6 \%$ (table 12.7). The quality and completeness of data have improved over the time that the annual audit has been running (table 12.8), although the number of centres providing data peaked in 2013. Completeness for some fields remained lower than $75 \%$ with access complications having particularly low levels of completion, although there is improvement here too.

Table 12.9 provides centre-level data for incident dialysis access, grouping patients by time of presentation to nephrology services (early $\geqslant 90$ or late $<90$ days before initiating dialysis). This table reproduces table 12.4 (which includes 2015 incident patients only) for incident dialysis patients between 2011 and 2015. Late presentation remains associated with low rates of definitive access placement compared with early presentation.

Table 12.10 provides an annual summary of rates of incident and prevalent definitive dialysis access and PTx. It shows that national performance from reporting centres has consistently fallen below Renal Association 
Table 12.7. Data completeness of the 2011-2015 annual Multisite Dialysis Access audits

\begin{tabular}{|c|c|c|c|c|c|}
\hline Centre and patient reporting & 2011 & 2012 & 2013 & 2014 & 2015 \\
\hline Centres reporting $(N)$ & 47 & 51 & 57 & 53 & 52 \\
\hline \multicolumn{6}{|l|}{ Incident HD patients included } \\
\hline$\%$ & 80.3 & 77.7 & 78.0 & 78.7 & 79.0 \\
\hline \multicolumn{6}{|l|}{ Incident PD patients included } \\
\hline$N$ & 839 & 1,008 & 1,041 & 1,069 & 1,075 \\
\hline Reported patients excluded as they did not match the UKRR data $(N)$ & 210 & 481 & 1,025 & 840 & 744 \\
\hline Reported patients excluded due to missing RRT start date or 1st access $(N)$ & 99 & 30 & 24 & 7 & 60 \\
\hline
\end{tabular}

HD - haemodialysis; PD - peritoneal dialysis; RRT - renal replacement therapy; UKRR - UK Renal Registry

Table 12.8. Percentage completeness of variables in the 2011-2015 annual Multisite Dialysis Access audits

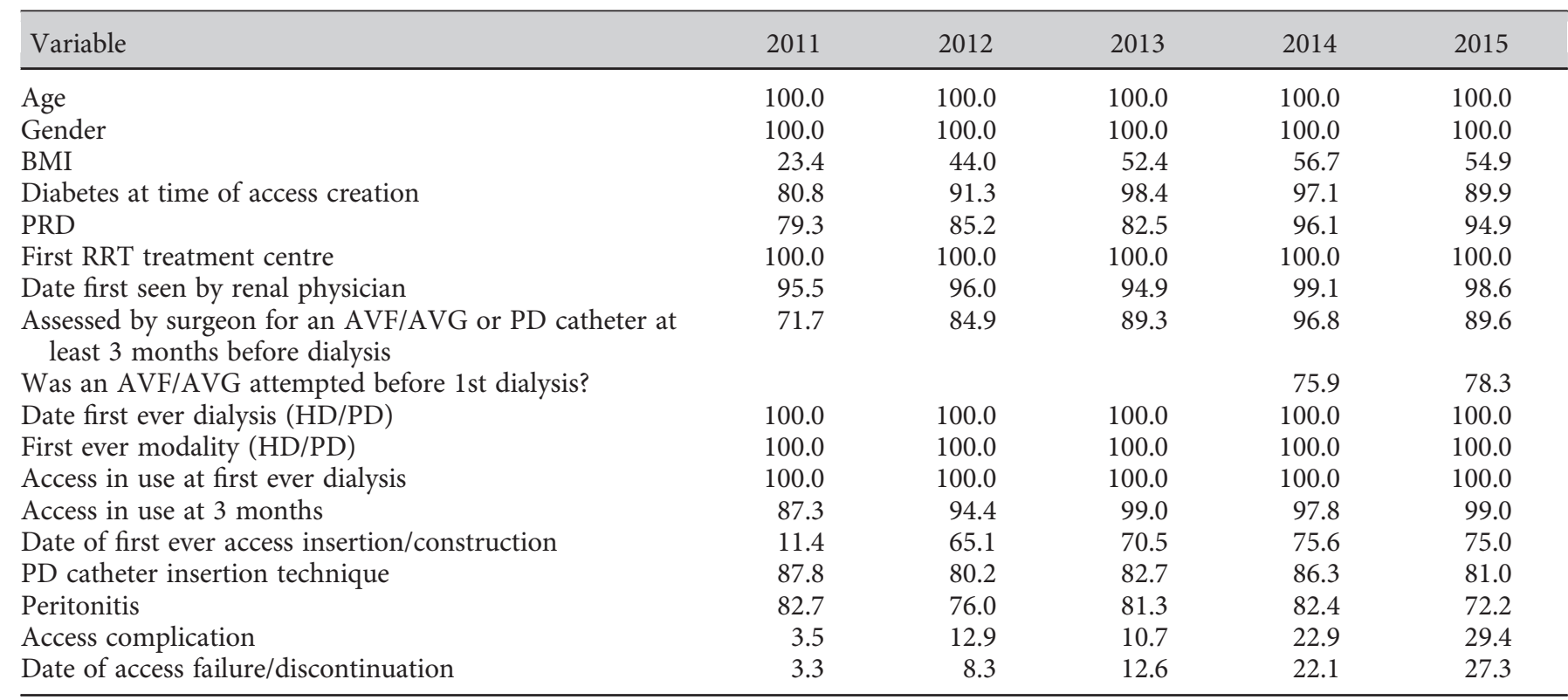

BMI - body mass index; PRD - primary renal diagnosis; HD - haemodialysis; PD - peritoneal dialysis; RRT - renal replacement therapy; AVF - arteriovenous fistula; AVG - arteriovenous graft

Table 12.9. Modality at start of dialysis and access, by referral time, by centre, including surgical referral rates, 2011-2015 data

\begin{tabular}{|c|c|c|c|c|c|c|c|c|c|c|c|c|c|c|c|}
\hline \multirow[b]{2}{*}{ Centre } & \multicolumn{5}{|c|}{ Early presenters ( $\geqslant 90$ days) $\%$} & \multicolumn{5}{|c|}{ Late presenters ( $<90$ days) \% } & \multicolumn{2}{|c|}{ Surg referral \% } & \multicolumn{3}{|c|}{ Treatment modality $N$} \\
\hline & $\mathrm{PD}$ & $\begin{array}{l}\text { AVF/ } \\
\text { AVG }\end{array}$ & $\mathrm{TL}$ & NTL & $\begin{array}{c}\text { Total } \\
N\end{array}$ & $\mathrm{PD}$ & $\begin{array}{l}\mathrm{AVF} / \\
\mathrm{AVG}\end{array}$ & $\mathrm{TL}$ & NTL & $\begin{array}{c}\text { Total } \\
N\end{array}$ & Yes & No & HD & $\mathrm{PD}$ & $\begin{array}{c}\text { Total } \\
N\end{array}$ \\
\hline Antrim & 20.2 & 47.7 & 12.8 & 19.3 & 109 & 5.3 & 0.0 & 31.6 & 63.2 & 19 & 68.0 & 32.0 & 111 & 24 & 135 \\
\hline B Heart & 19.5 & 48.5 & 16.0 & 16.0 & 462 & 11.8 & 23.5 & 23.5 & 41.2 & 17 & 48.9 & 51.1 & 407 & 96 & 503 \\
\hline $\mathrm{B}$ QEH & 24.0 & 42.9 & 32.7 & 0.4 & 741 & 9.1 & 8.6 & 82.3 & 0.0 & 186 & 65.0 & 35.0 & 797 & 207 & 1,004 \\
\hline Bangor & 26.5 & 34.7 & 29.6 & 9.2 & 98 & 10.0 & 10.0 & 60.0 & 20.0 & 10 & 67.9 & 32.1 & 83 & 27 & 110 \\
\hline Basldn & 26.7 & 44.3 & 19.1 & 9.9 & 131 & 10.5 & 5.3 & 10.5 & 73.7 & 19 & 65.4 & 34.6 & 121 & 38 & 159 \\
\hline Belfast & 17.6 & 32.0 & 18.9 & 31.6 & 244 & 1.8 & 0.0 & 9.1 & 89.1 & 55 & 51.9 & 48.1 & 267 & 46 & 313 \\
\hline Bradfd & 15.4 & 43.8 & 30.5 & 10.3 & 272 & 5.6 & 8.3 & 41.7 & 44.4 & 36 & 52.8 & 47.2 & 274 & 45 & 319 \\
\hline Brightn & 27.8 & 35.8 & 22.6 & 13.8 & 486 & 12.4 & 4.4 & 22.1 & 61.1 & 113 & 39.9 & 60.1 & 479 & 153 & 632 \\
\hline Bristol & 19.3 & 56.0 & 20.0 & 4.7 & 150 & 6.7 & 6.7 & 46.7 & 40.0 & 15 & 85.8 & 14.2 & 205 & 37 & 242 \\
\hline
\end{tabular}


Table 12.9. Continued

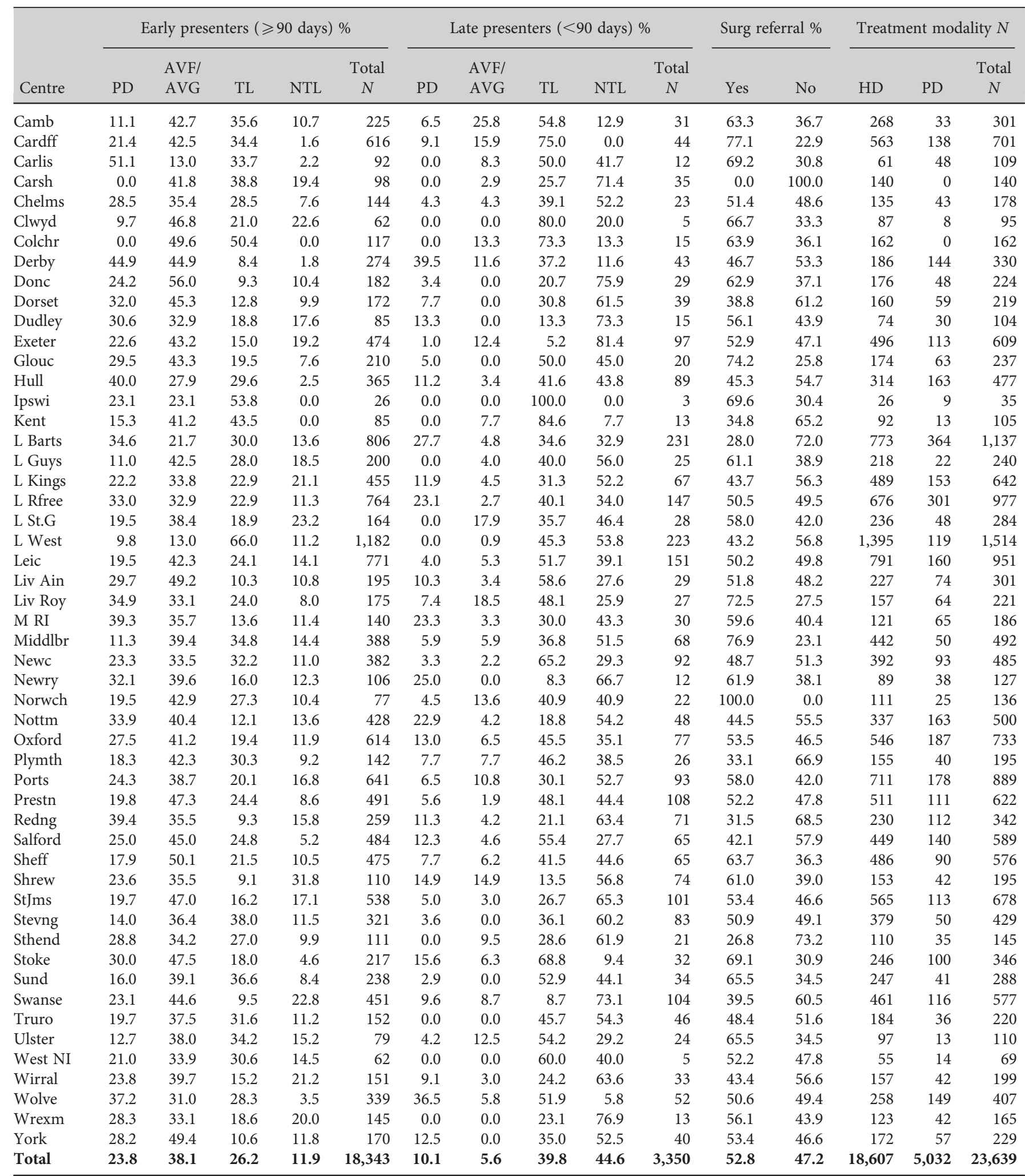

Surg - surgical; PD - peritoneal dialysis; AVF - arteriovenous fistula; AVG - arteriovenous graft; TL - tunnelled line; NTL - non-tunnelled line 
Table 12.10. Annual rates of definitive access and pre-emptive transplantation and concordance with Renal Association audit standards

\begin{tabular}{|c|c|c|c|c|c|c|}
\hline Modality in incident and prevalent patients & 2011 & 2012 & 2013 & 2014 & 2015 & Audit standard \\
\hline \multicolumn{7}{|l|}{ Incident patients } \\
\hline Definitive access (AVF/AVG/PD) or PTx in incident RRT patients (\%) & 56.5 & 57.6 & 58.7 & 56.5 & 55.3 & None set \\
\hline $\begin{array}{l}\text { Definitive access (AVF/AVG/PD) or PTx in incident RRT patients excluding } \\
\text { late presenters }(\%)\end{array}$ & 62.9 & 63.4 & 64.8 & 62.6 & 61.4 & None set \\
\hline AVF/AVG in incident HD patients (\%) & 41.4 & 40.9 & 41.8 & 39.2 & 38.6 & None set \\
\hline AVF/AVG in incident HD patients, excluding late presentation (\%) & 48.3 & 47.4 & 48.4 & 45.6 & 45.2 & $60 \%$ \\
\hline AVF/AVG/PD in incident dialysis patients (\%) & 53.0 & 54.0 & 54.6 & 52.2 & 51.7 & None set \\
\hline AVF/AVG/PD in incident dialysis patients excluding late presentation (\%) & 59.5 & 59.9 & 60.8 & 58.3 & 57.8 & None set \\
\hline \multicolumn{7}{|l|}{ Prevalent patients } \\
\hline Definitive access (AVF/AVG/PD) in prevalent dialysis patients (\%) & * & 82.3 & 79.0 & 73.6 & 72.6 & $80 \%$ \\
\hline AVF/AVG in prevalent HD patients (\%) & * & 79.0 & 75.4 & 69.6 & 68.6 & None set \\
\hline
\end{tabular}

In 2015, audit standards were updated for AVF/AVG in incident HD patients (minimum standard reduced from 65\% to 60\%); incident RRT recipients (to include PD and PTx; no minimum standard set) and prevalent dialysis patients (to include PD and HD via AVF/AVG 'definitive access', minimum standard $80 \%$ ). It is not entirely the same centres submitting access data each year and therefore direct year-toyear comparisons in performance are not valid.

HD - haemodialysis; PD - peritoneal dialysis; AVF - arteriovenous fistula; AVG - arteriovenous graft; TL - tunnelled line; NTL - nontunnelled line; RRT - renal replacement therapy

*Prevalent data were not collected in the 2011 audit

minimum guideline standards. Direct year-to-year comparisons in performance are not valid due to annual changes in centres providing data and quality of data completion. A secondary analysis including only centres that have reported continuously from 2012 to 2015 (data not shown), shows that for these centres the trend was similar to the results in table 12.10 .

Table 12.11 shows dialysis access three months after initiation, stratified by first access used for patients from the 2011-2015 audits. This reproduces table 12.6 (which includes 2015 patients only). As before, the majority $(85.8 \%)$ of patients who initiated with definitive access continued with definitive access or had been transplanted at three months. Of patients who started dialysis without definitive access, $28.4 \%$ received a transplant or were dialysing with definitive access at three months.
Death before three months was much more common in patients initiating dialysis with a NTL than with any other form of initial access (12.7\%), which is lower than the 2015 data (22.4\%).

Figures 12.20-12.25 replicate figures 12.2-12.7 (2015 incident patients only) to include all incident patients from 2011-2015. The trends described in figures 12.212.7 are largely reproduced. Data completeness for BMI and diabetic status remained low with multiple centrelevel exclusions.

Figure 12.26 plots the incident RRT approach stratified by centre and reproduces figure 12.8 (2015 data only) for incident RRT patients from 2011-2015. The most notable feature is centre-to-centre variation in rates of PD as an incident modality. Use of PTx appears less strongly associated with centre size than for 2015 data.

Table 12.11. Type of dialysis access at 90 days stratified by initial modality in 2011-2015

\begin{tabular}{|c|c|c|c|c|c|c|c|c|c|}
\hline \multirow{2}{*}{$\begin{array}{l}\text { Access in use at } \\
\text { first dialysis }(N)\end{array}$} & \multicolumn{9}{|c|}{ Access in use at three months (\%) } \\
\hline & AVF/AVG & $\mathrm{TL}$ & NTL & PD catheter & $\mathrm{Tx}$ & Died & Stop/LTFU & Recovered & Missing \\
\hline TL $(6,900)$ & 10.7 & 75.8 & 0.3 & 2.7 & 1.0 & 5.8 & 0.5 & 0.0 & 3.3 \\
\hline NTL $(4,213)$ & 8.2 & 64.1 & 4.7 & 5.5 & 0.3 & 12.7 & 0.8 & 0.0 & 3.7 \\
\hline
\end{tabular}

Shaded cells highlight the percentage of patients who remained on the same modality at three months PD - peritoneal dialysis; AVF - arteriovenous fistula; AVG - arteriovenous graft; Tx - transplant; TL - tunnelled line; NTL - non-tunnelled line; LTFU - lost to follow-up 

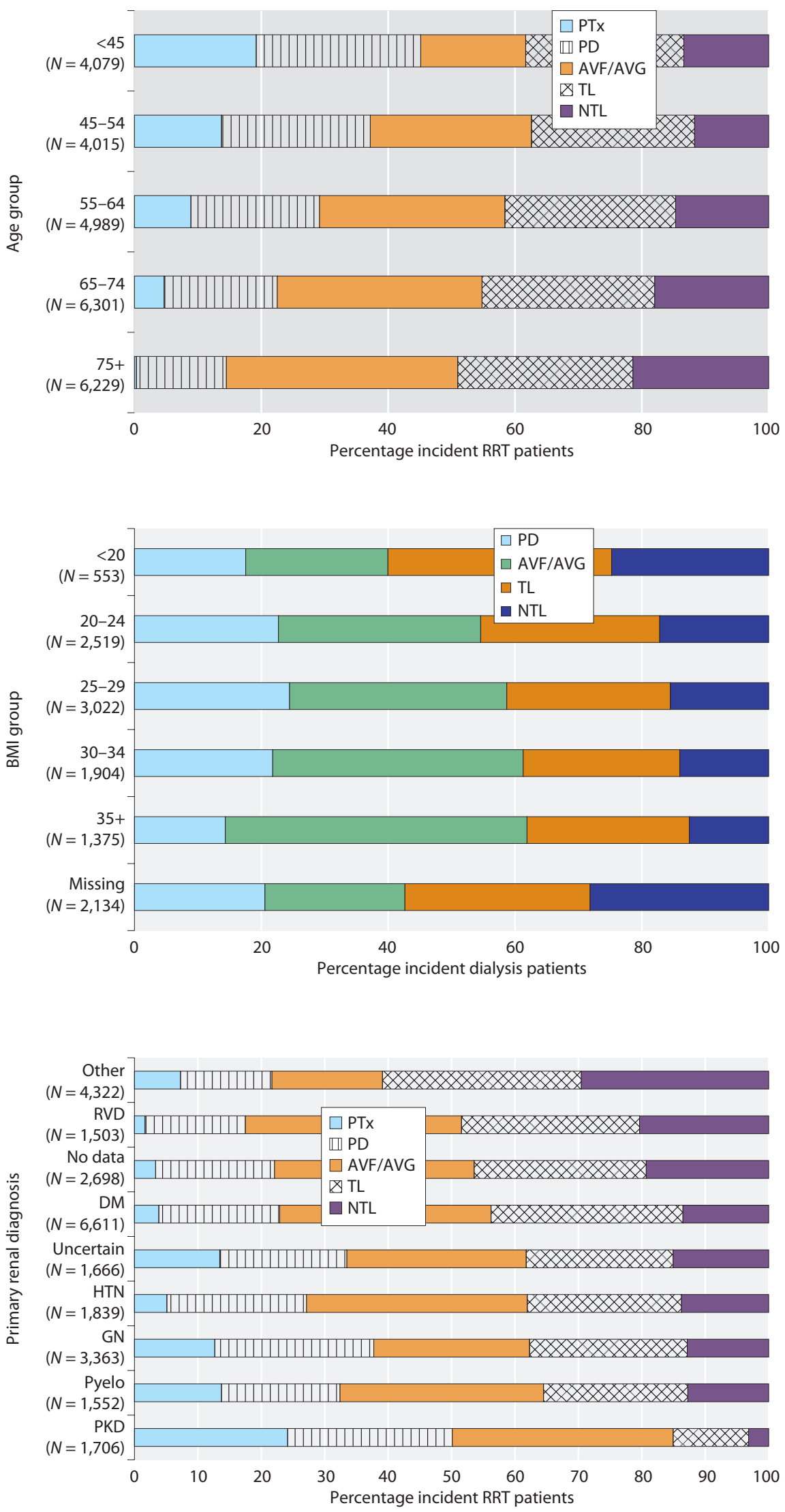

Fig. 12.20. Percentage of incident RRT patients stratified by age and access at start, 2011-2015

Number of patients in each group in brackets. PTx - pre-emptive transplant; PD - peritoneal dialysis; AVF - arteriovenous fistula;

AVG - arteriovenous graft; TL - tunnelled line; NTL - non-tunnelled line

Fig. 12.21. Percentage of incident dialysis patients stratified by BMI and access at start, 2011-2015

Number of patients in each group in brackets. PD - peritoneal dialysis; AVF - arteriovenous fistula; AVG - arteriovenous graft; TL - tunnelled line; NTL - non-tunnelled line; BMI - body mass index
Fig. 12.22. Percentage of incident RRT patients stratified by PRD and access at start, 2011-2015

Number of patients in each group in brackets. PRD groups are sorted by decreasing proportion of patients initiating RRT with a HD catheter. PRD - primary renal diagnosis; DM - diabetes mellitus; GN - glomerulonephritis; HTN hypertension; PKD - polycystic kidney disease; Pyelo - pyelonephritis; RVD - renal vascular disease

PTx - pre-emptive transplant; PD - peritoneal dialysis; AVF - arteriovenous fistula;

AVG - arteriovenous graft; TL - tunnelled line; NTL - non-tunnelled line 

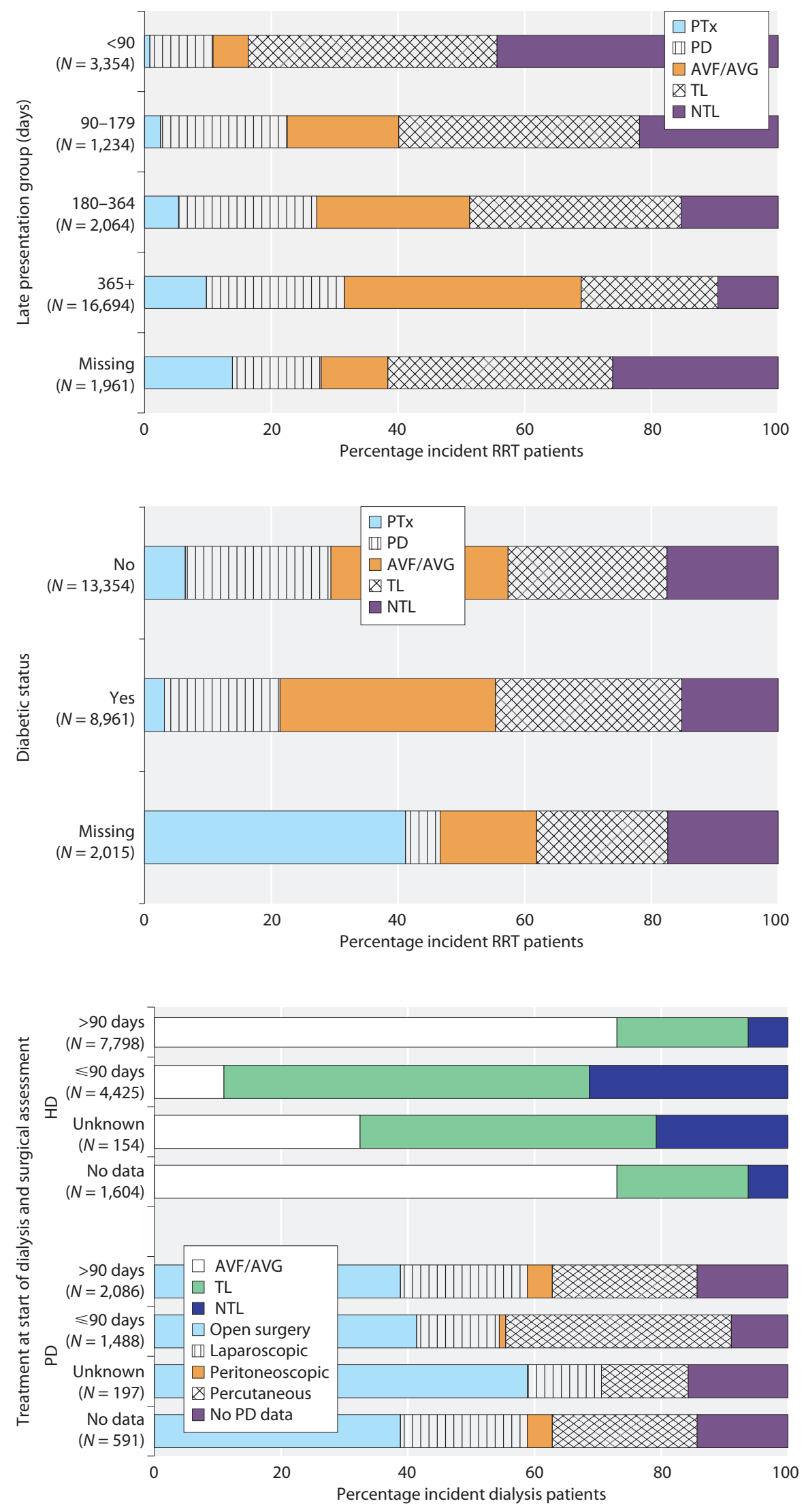

Fig. 12.23. Percentage of incident RRT patients stratified by length of time known to nephrology and access at start, 2011-2015 Number of patients in each group in brackets. PTx - pre-emptive transplant; PD - peritoneal dialysis; AVF - arteriovenous fistula;

AVG - arteriovenous graft; TL - tunnelled line; NTL - non-tunnelled line

Fig. 12.24. Percentage of incident RRT patients by diabetic status and access at start, 2011-2015

Number of patients in each group in brackets. PTx - pre-emptive transplant; PD - peritoneal dialysis; AVF - arteriovenous fistula;

AVG - arteriovenous graft; TL - tunnelled line; NTL - non-tunnelled line

Fig. 12.25. Percentage of incident dialysis patients by assessment by a surgeon within 3 months before starting RRT and access at start of dialysis, 2011-2015

Number of patients in each group in brackets. Late presenting patients were excluded from the analysis.

AVF - arteriovenous fistula; AVG -

arteriovenous graft; TL - tunnelled line; NTL non-tunnelled line; PD - peritoneal dialysis

Hole/Caskey/Evans/Fluck/Kumwenda/ Steenkamp/Wilkie 


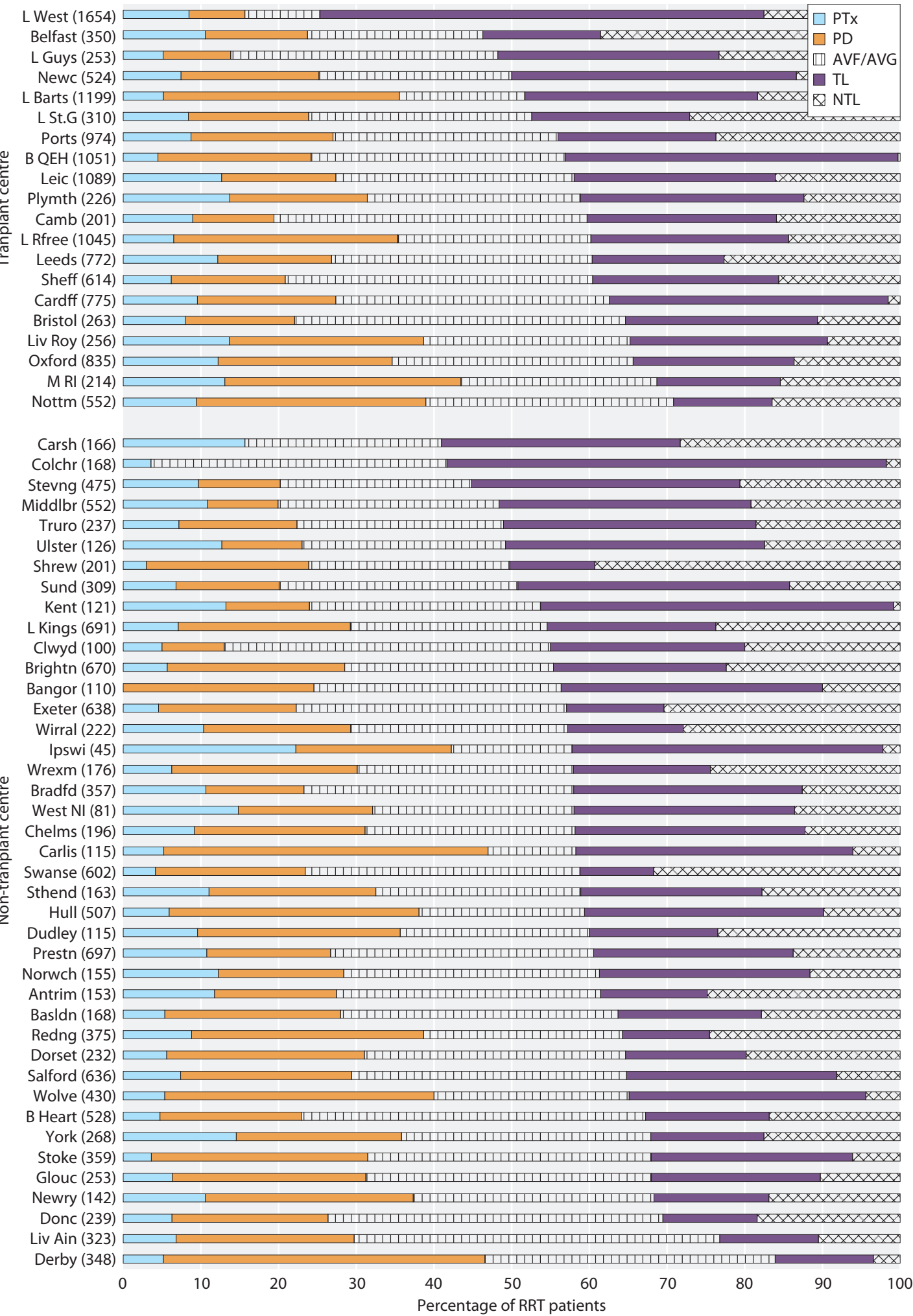

Fig. 12.26. Incident RRT approach for patients in the 2011-2015 Multisite Dialysis Access audits, stratified by renal centre Centre size (patient numbers) in brackets. Centres are stratified by transplant/non-transplant centre and sorted by proportion of patients initiating HD with a TL/NTL. PTx - pre-emptive transplant; PD - peritoneal dialysis; AVF - arteriovenous fistula; AVG - arteriovenous graft; TL - tunnelled line; NTL - non-tunnelled line; RRT - renal replacement therapy 


\section{Discussion}

In this fifth annual multisite dialysis access audit, information is presented on the nature, timeliness and durability of initial dialysis access for 23,639 incident RRT patients. This accounts for $71.6 \%$ of patients starting dialysis in England, Wales and Northern Ireland over this period. These data describe national and centre-level performance and identify patient and system factors that are associated with practice patterns. The centres contributing data to the audit have changed, so it is not appropriate to make direct year-to-year comparisons. However, definitive access amongst both incident and prevalent patients was below Renal Association audit standards for nearly every year of the data collection. There were a small number of centres achieving high rates of definitive dialysis access for incident and prevalent dialysis recipients, demonstrating that the audit standards are attainable. In addition, the Dialysis Outcomes and Practice Patterns Study (DOPPS) suggests that the UK is improving in overall performance [3]. A better understanding of the practice patterns at high performing centres has the potential to provide information to inform wider quality improvement.

This audit confirms that timely presentation to a nephrologist and referral to a dialysis access surgeon are associated with higher rates of definitive dialysis access use. Most patients who only meet a nephrologist for the first time within three months of starting dialysis commenced HD via a NTL/TL. However, a substantial proportion of patients known to a nephrologist for more than three months also commenced HD via this form of access, and indeed conversion from a NTL/TL to definitive access by three months was infrequent in most centres. The need to begin access planning early is confirmed by the observation that $86.3 \%$ of individuals who had access attempted more than a year before initiating HD, started with an AVF/AVG. A small number of centres were however, able to secure definitive access within three months, achieved in part by promoting the use of PD. Most commonly, responsive PD access pathways were achieved through the use of the percutaneous rather than surgical catheter insertion pathways. This is logical, since this approach is generally performed under local anaesthetic, avoiding the requirement for both scheduling a pre-operative assessment and operating theatre time. No evidence was found in this audit to suggest percutaneous placement of PD catheters was inferior to surgical placement, since catheter function at one year was similar for all insertion techniques. A number of centres were able to achieve rapid surgical pathways for vascular access. Again, efforts to better understand practice patterns that enhance the responsiveness of vascular and PD access services are needed. Results from the UK Peritoneal Dialysis Outcomes and Practice Patterns Study (PDOPPS) Catheter Study [4] and a national survey of $\mathrm{HD}$ access in the UK by the British Renal Society Vascular Access Special Interest Group will inform practice.

It has been argued that lower rates of definitive dialysis access in some centres may be a result of higher rates of PTx, because these patients may otherwise have started dialysis with definitive access. For this reason, PTx data are included alongside the dialysis access data in some places and allocated patients to centres according to the catchment area of the dialysis centre, rather than the transplant centre that they first appeared in. These data were therefore provided to explore the impact of preemptive and early transplant on dialysis access rates and were not intended as a study of variation in rates of transplantation. Importantly, there was no strong evidence to demonstrate that definitive dialysis access use was influenced by transplant/non-transplant centre status, or by the proportion of patients receiving PTx at centre level. Previous versions of this chapter have noted counterintuitive associations between increasing age and BMI with AVF/AVG use. The increased proportional use of $A V F / A V G$ with increasing age and BMI reflect the lower use of $\mathrm{PD}$ and transplantation amongst older people and the obese. It is presumably for the same reason that the proportional use of TL/NTL amongst incident RRT recipients increased with age and BMI. Inclusion of PTx data has also highlighted the prominent differences in practice patterns between primary renal diagnoses. For example, rates of PTx and definitive access were particularly high for people with polycystic kidney disease. People with this primary diagnosis were likely to be known to nephrologists for several years prior to starting RRT and to have enhanced health literacy due to the familial nature of the condition - both factors that increase the opportunity for preparation for RRT. Understanding the factors that contribute to success in this group may allow effective components of the access pathway to be disseminated. Further unexplained patterns remain that require exploration, such as lower rates of AVF/AVG use amongst individuals whose ethnicity was listed as Black and the low use of definitive access in patients with BMI $<20$.

The UKRR has an important role in monitoring the quality of planned and unplanned RRT provision and 
informing guidance and practice improvement. Centrelevel data are provided as a surrogate of geographical variation in RRT provision. Wide variation in practice reflects the absence of a cohesive approach, despite national guidance. The insights gained from the inclusion of all information about all three RRT modalities in this chapter reflect the importance of a comprehensive approach in the exploration of trends in RRT access provision. Once again, this year's multisite dialysis access audit identifies the need for research and quality improvement initiatives to enhance dialysis access practice. The following approaches may help to generate the knowledge required to drive this process:

- Detailed practice pattern assessment of high and low-performing centres and those that have demonstrated marked improvement in their delivery of definitive access.
- Assessment of responsive pathways to PD access formation, with particular focus on the role of surgical and non-surgical insertion technique and treatment pathways that facilitate initiation of PD within 90 days.

- Use of UKRR data to analyse the associations between dialysis access at initiation and outcomes beyond one year, including dialysis catheter-related complications.

- Improvement in the completeness of data provision for the annual multisite dialysis access audit.

\section{Acknowledgements}

Thanks are expressed to all renal centres for their assistance in providing the data.

Conflicts of interest: the authors declare no conflicts of interest

\section{References}

1 Fluck R, Pitcher D and Steenkamp R. Vascular Access Audit Report 2012 UK Renal Registry and NHS Kidney Care, 2012. Available from: https:// www.renalreg.org/documents/vascular-access-audit-report/.

2 Judge, A., et al., Inequalities in rates of renal replacement therapy in England: does it matter who you are or where you live? Nephrol Dial Transplant, 2012. 27(4): p. 1598-1607.

3 The Dialysis Outcomes and Practice Patterns Study. Available from: http://www.dopps.org/OurStudies/HemodialysisDOPPS.aspx.

4 Briggs, V., et al., UK Catheter Study - Protocol Synopsis. Peritoneal Dialysis International. Accepted for publication. 
